\title{
Nebular abundances of nearby southern dwarf galaxies
}

\author{
Henry Lee ${ }^{1,2, \star}$, E. K. Grebel ${ }^{1}$, and P. W. Hodge ${ }^{3}$ \\ 1 Max-Planck-Institut für Astronomie, Königstuhl 17, 69117 Heidelberg, Germany \\ 2 Dept. of Physics \& Astronomy, York University, 4700 Keele St., Toronto, Ontario M3J 1P3, Canada \\ 3 Astronomy Department, University of Washington, Box 351580, Seattle, WA 98195-1580, USA
}

Received 30 August 2002 / Accepted 15 January 2003

\begin{abstract}
The results of optical spectroscopy of H II regions in a sample of southern dwarf irregulars consisting of five dwarf galaxies in the Centaurus A group, four dwarfs in the Sculptor group, and eight additional dwarf galaxies are presented. Oxygen abundances are derived using the direct method where [O III] $\lambda 4363$ is detected; otherwise, abundances are derived with the bright-line method using the McGaugh and the Pilyugin calibrations. ESO358-G060 has the lowest oxygen abundance $(12+\log (\mathrm{O} / \mathrm{H})=7.32)$ in the sample, which is comparable to the value for the second most metal-poor galaxy known (SBS 0335-052). In all, new oxygen abundances are reported for nine dwarf galaxies; updated values are presented for the remaining galaxies. Derived oxygen abundances are in the range from $3 \%$ to $26 \%$ of the solar value. Oxygen abundances for dwarfs in the southern sample are consistent with the metallicity-luminosity relationship defined by a control sample of dwarf irregulars with [O III] $\lambda 4363$ abundances and well-measured distances. However, NGC 5264 appears to have an (upper branch) oxygen abundance approximately two to three times higher than other dwarfs at similar luminosities. Nitrogen-to-oxygen and neon-to-oxygen abundance ratios are also reported; in particular, IC 1613 and IC 5152 show elevated nitrogen-to-oxygen ratios for their oxygen abundances.
\end{abstract}

Key words. galaxies: abundances - galaxies: clusters: individual: Centaurus A, Sculptor - galaxies: dwarf galaxies: evolution - galaxies: irregular

\section{Introduction}

Dwarf galaxies, the most abundant type of galaxy in groups and clusters, may hold the key to understanding galaxy evolution. In standard hierarchical structure formation scenarios, they are the building blocks of more massive galaxies. Conversely, the environment - e.g., the presence or absence of more massive galaxies in the vicinity - surrounding dwarf galaxies is expected to affect their evolution (e.g., Mayer et al. 2001). Furthermore, the intrinsic properties of a dwarf galaxy - e.g., its mass, density, gas content - are likely to influence the histories of star formation and chemical enrichment. However, the relative importance of external and internal properties and their actual effect on dwarf galaxy evolution remains unknown. The most detailed studies of dwarf galaxy properties have been carried out in the Local Group, but the limited sample size and the wide range of properties prevent us from obtaining a unified picture.

To arrive at a better understanding of what drives dwarf galaxy evolution we are carrying out a comprehensive

Send offprint requests to: H. Lee, e-mail: lee@mpia.de

^ Visiting Astronomer, Cerro Tololo Inter-American Observatory, National Optical Astronomy Observatories, operated by the Association of Universities for Research in Astronomy, Inc. (AURA) under cooperative agreement with the National Science Foundation. multi-wavelength study of dwarf galaxies in the Local Volume ( $\$ 5 \mathrm{Mpc}$ ). The Local Volume contains several nearby galaxy groups as well as galaxies in the relative isolation of the field. After the conclusion of an all-sky survey to detect dwarf galaxy candidates in the Local Volume (Karachentsev et al. 2000, and references therein) we are carrying out ground-based imaging to derive integrated photometry and structural parameters (e.g., Makarova et al. 2002). These efforts are complemented by two snapshot programs with the Hubble Space Telescope's (HST) Wide Field and Planetary Camera 2 (WFPC2) totalling 200 orbits to study stellar content, recent star formation histories, distances, and relative positions within galaxy groups (e.g., Karachentsev et al. 2002a,b,c). Radio observations (e.g., Huchtmeier et al. 2000a,b) reveal the HI content and radial velocity of the dwarfs, provided that they contain detectable amounts of H I. For a description of the project as a whole, see Grebel et al. (2000).

Since our HST data enable us to measure relative distances within galaxy groups, we are in the unique position of being able to assign membership probabilities to individual dwarf galaxies and to consider them based on their three-dimensional location within a group. Our ground-based imaging in combination with distances yields information on the luminous mass of dwarf galaxies. Compiling a database that, in addition, contains accurate information on chemical abundances will allow 
us to consider dwarf galaxies within their evolutionary context in groups and in the field. The knowledge of relative distances and intrinsic properties is a major step towards resolving what governs dwarf galaxy evolution.

The data presented here are part of our continuing efforts to obtain abundances for dwarf irregular galaxies (dIs) in the Local Volume and are based on the first two observing runs dedicated to this multi-year project. However, not all targets have HST-based distances. At the time of writing, about 150 out of the 200 dwarf galaxy candidates in the Local Volume have been observed, as we await the completion of the HST snapshot survey. Galaxies were selected according to the following criteria: (a) galaxies were visible at the epoch of observations, and (b) galaxies were sufficiently luminous to be observable with a $1.5-\mathrm{m}$ class telescope. Hence, the present study consists primarily of results for individual galaxies and of the discussion for these galaxies in the metallicityluminosity relationship. The solar value of the oxygen abundance of $12+\log (\mathrm{O} / \mathrm{H})=8.87$ (Grevesse et al. 1996) is adopted for the present work to facilitate comparisons with earlier studies. However, recent work has indicated that the solar value may in fact be smaller by about 0.1 to $0.2 \mathrm{dex}$ (Allende Prieto et al. 2001; Holweger 2001).

The outline of this paper is as follows. A brief description of the observed galaxies is presented in Sect. 2. Observations and reductions of the data are presented in Sect. 3. Measurements and analysis are discussed in Sect. 4, and nebular abundances are presented in Sect. 5. A discussion of individual galaxies and of the metallicity-luminosity relation is provided in Sect. 6. A summary is given in Sect. 7.

\section{The samples of dwarf galaxies}

\subsection{The southern sample of dls}

The southern sample consists of five dwarf galaxies from the Centaurus A (NGC 5128, Cen A) group, four dwarf galaxies from the Sculptor ( $\mathrm{Scl})$ group, one dwarf in the AntliaSextans group, and seven dwarf galaxies in the Local Group and in the field. In particular, IC 1613, IC 5152, NGC 2915, and NGC 3109 were chosen because oxygen abundances in the literature were derived from measurements using obsolete technology or abundances were not well measured. Two galaxies (A0355-465, IC 2032) in the field were selected from the list compiled by Karachentseva \& Karachentsev (1998), and two galaxies (ESO358-G060, ESO302-G014) were selected from the Feitzinger \& Galinski (1985) catalog. ESO358-G060 is a member of the Fornax Cluster; see Sect. 6.1.10 for additional details.

Because half of the galaxies are members of two nearby groups of galaxies, a brief summary of prior spectroscopy is mentioned here. The Centaurus A group and the Sculptor group are each at a distance of $\$ 5 \mathrm{Mpc}$. Webster \& Smith (1983) and Webster et al. (1983) obtained oxygen abundances for southern irregular and spiral galaxies, including galaxies in the Cen A group and in the field, with a measured range of abundances from about ten to sixty per cent of the solar value. Miller (1996) carried out $\mathrm{H} \alpha$ imaging of eight
Sculptor group dwarfs; only two contained detectable H II regions. Subsequent H II region spectroscopy of these two dwarfs showed that their oxygen abundances were roughly 0.1 of the solar value, although the temperature-sensitive [O III] $\lambda 4363$ emission line (see Sect. 5) was not detected in either galaxy. Hidalgo-Gámez et al. (2001) measured [O III] 24363 in the low surface brightness dI ESO245-G005 in the Scl group. [O III] $\lambda 4363$ was detected in two H II regions; however, the two resulting oxygen abundances differ by 0.21 dex, which may be evidence of an abundance gradient. This is similar to the gradient $\left(\approx 0.2 \mathrm{dex} \mathrm{kpc}^{-1}\right)$ from spectra of seven H II regions for the same galaxy measured by Miller (1996), although he determined oxygen abundances using the indirect or brightline method (see Sect. 5.2).

Properties of galaxies in the southern sample are listed in Table 1. Galaxies with probable Local Group membership are listed in Grebel (1999, 2000), and Grebel et al. (2003). Compilations of oxygen abundances for nearby dwarf irregulars are found in, e.g., Mateo (1998), Pilyugin (2001b), and Lee et al. (2003a). Comments about individual galaxies from the southern sample will be addressed in Sect. 6.1.

\subsection{The control sample of dls}

Richer \& McCall (1995) constructed a sample of nearby dIs with direct ([O III] 44363 ) oxygen abundances and wellmeasured distances from resolved stellar photometry. As a part of his thesis, Lee updated the sample incorporating updates from recent literature and unpublished spectroscopic data (Lee 2001; Lee et al. 2003a). This sample of dIs will be referred to as the control sample, against which the present sample of southern dwarfs will be compared. Because new measurements of IC 1613 and NGC 3109 are discussed here, they are excluded from the control sample in this paper and are included in the present southern sample of dIs.

As part of a growing sample of galaxies which satisfy the criteria above, UGC 4483 is added here to the list of dIs in the control sample. Direct oxygen and nitrogen abundances were measured by Skillman et al. (1994) and Izotov et al. (1994). Average values of $12+\log (\mathrm{O} / \mathrm{H})=7.53$ and $\log (\mathrm{N} / \mathrm{O})=-1.60$ are adopted here. Tip of the red giant branch (TRGB) distances were obtained independently by Dolphin et al. (2001a) and Izotov \& Thuan (2002); an average distance of $3.3 \mathrm{Mpc}$ $(m-M=27.59)$ is adopted here. The integrated 21-cm flux integral is $13.34 \mathrm{Jy} \mathrm{km} \mathrm{s}^{-1}$ (van Zee et al. 1998a), which gives $M_{\mathrm{HI}}=3.42 \times 10^{7} M_{\odot}$ and $M_{\mathrm{HI}} / L_{B}=1.96 M_{\odot} / L_{\odot, B}$.

\section{Observations and reductions}

Long-slit spectroscopic observations were carried out in two observing runs (2001 Feb. 2-4 UT and Aug. 17-20 UT) with the Cassegrain Spectrograph on the 1.5-metre telescope at the Cerro Tololo Inter-American Observatory. Details of the instrumentation employed and the log of observations are listed in Tables 2 and 3, respectively. Observing conditions were varied. For the February run, all three nights were clear, although high thin patchy clouds were present at times. The lunar illumination increased from 0.55 to 0.76 . For the August run, cloud 
Table 1. Basic properties of galaxies in the southern sample. Galaxies are grouped by association to a group (or otherwise) and are listed in alphabetical order within each category. All properties are obtained from NED, unless otherwise noted. Columns (1) and (2): Galaxy name used in the present work; other names. Column (3): Membership. Column (4): Classification type. Column (5): Heliocentric velocity. Column (6): Total apparent $B$ magnitude. Column (7): Total H I 21-cm flux. Rotational velocities in H I have been measured for A1243-335, A1334-277, DDO 161, IC 1613, and UGCA 442 (Hoffman et al. 1996; Côté et al. 2000); a rotation curve in H $\alpha$ for ESO358-G060 has been measured by Matthews \& Gallagher (2002). Column (8): Total H I mass to blue luminosity ratio. Column (9): Measured or estimated distances. Centaurus A group galaxies: Karachentsev et al. (2002b), except DDO 161 (Côté et al. 2000). Sculptor group galaxies: Côté et al. (2000), except UGCA 442 (Karachentsev et al. 2003b). Other southern galaxies: ESO358-G060 - Mould et al. (2000); IC 1613 - Dolphin et al. (2001b); IC 2032 Carrasco et al. (2001); NGC 2915 - Karachentsev et al. (2003a); NGC 3109, Sag DIG, and IC 5152 - Karachentsev et al. (2002c). NOTES: ${ }^{a}$ From Côté et al. (1997). ${ }^{b}$ From Longmore et al. (1982). ${ }^{c}$ Velocity estimated from spectrum (Sect. 6.1). ${ }^{d}$ From Barnes et al. (1997). ${ }^{e}$ Member of Fornax Cluster Catalog (FCC; Ferguson 1989). ${ }^{f}$ From Schröder et al. (2001); see also Matthews et al. (1998). ${ }^{g}$ From Hoffman et al. (1996). ${ }^{h}$ From Huchtmeier et al. (2000a). ${ }^{i}$ From Huchtmeier \& Richter (1986). ${ }^{j}$ From Meurer et al. (1996). ${ }^{k}$ Common group of dwarfs including NGC 3109, Sextans A, Sextans B, and the Antlia dwarf (van den Bergh 1999; Tully et al. 2002). ${ }^{l}$ From Barnes \& de Blok (2001). ${ }^{m}$ From Lee \& Kim (2000). ${ }^{n}$ From Young \& Lo (1997).

\begin{tabular}{|c|c|c|c|c|c|c|c|c|}
\hline $\begin{array}{l}\text { Galaxy } \\
\text { (1) }\end{array}$ & $\begin{array}{c}\text { Other } \\
\text { Name(s) } \\
(2)\end{array}$ & $\begin{array}{c}\text { Member } \\
\text { (3) }\end{array}$ & $\begin{array}{l}\text { Type } \\
\text { (4) }\end{array}$ & $\begin{array}{c}v_{\odot} \\
\left(\mathrm{km} \mathrm{s}^{-1}\right) \\
(5)\end{array}$ & $\begin{array}{c}B_{T} \\
(\mathrm{mag}) \\
(6)\end{array}$ & $\begin{array}{c}F_{21} \\
\left(\mathrm{Jy} \mathrm{km} \mathrm{s}^{-1}\right) \\
(7)\end{array}$ & $\begin{array}{c}M_{\mathrm{HI}} / L_{B} \\
\left(M_{\odot} / L_{\odot, B}\right) \\
(8)\end{array}$ & $\begin{array}{c}D \\
(\mathrm{Mpc}) \\
(9)\end{array}$ \\
\hline \multicolumn{9}{|c|}{ Centaurus A group dwarfs } \\
\hline A1243-335 & ESO381-G020, AM1243-333 & Cen A & $\mathrm{IB}(\mathrm{s}) \mathrm{m}$ & +585 & 14.24 & $31.9^{a}$ & 1.85 & 3.63 \\
\hline A1324-412 & ESO324-G024, AM1324-411 & Cen A & $\mathrm{IAB}(\mathrm{s}) \mathrm{m}$ : & +513 & 12.91 & $52.1^{a}$ & 0.75 & 3.73 \\
\hline A1334-277 & ESO444-G084, AM1334-274 & Cen A & Im & +587 & 15.01 & $19.6^{a}$ & 2.29 & 4.61 \\
\hline A1346-358 & ESO383-G087, AM1346-354 & Cen A & $\mathrm{SB}(\mathrm{s}) \mathrm{dm}$ & +326 & 11.00 & $27.4^{a}$ & 0.08 & 3.63 \\
\hline DDO 161 & UGCA 320, A $1300-17$ & Cen A & $\mathrm{IB}(\mathrm{s}) \mathrm{m} \mathrm{sp}$ & +744 & 13.52 & $110.1^{a}$ & 3.10 & 5.25 \\
\hline NGC 5264 & DDO 242, UGCA 370 & Cen A & $\mathrm{IB}(\mathrm{s}) \mathrm{m}$ & +478 & 12.60 & $13.7^{a}$ & 0.19 & 4.53 \\
\hline \multicolumn{9}{|c|}{ Sculptor group dwarfs } \\
\hline AM0106-382 & $\ldots$ & $\mathrm{Scl}$ & dwarf Im & +645 & 16.26 & $<2.8^{a}$ & $<1.29$ & 3.0 \\
\hline ESO347-G017 & PGC071466 & Scl & $\mathrm{SB}(\mathrm{s}) \mathrm{m}:$ & +659 & 14.19 & $10.5^{a}$ & 0.71 & 3.0 \\
\hline ESO348-G009 & A2346-380, AM2346-380 & $\mathrm{Scl}$ & $\mathrm{IBm}$ & +657 & $13.60^{b}$ & $8.4^{a}$ & 0.33 & 3.0 \\
\hline UGCA 442 & ESO471-G006, AM2341-321 & $\mathrm{Scl}$ & $\mathrm{SB}(\mathrm{s}) \mathrm{m}:$ & +267 & 13.60 & $54.3^{a}$ & 2.12 & 4.27 \\
\hline \multicolumn{9}{|c|}{ Other southern dwarfs } \\
\hline $\mathrm{A} 0355-465$ & ESO249-G032, AM0355-463 & field & $\mathrm{IB}(\mathrm{s}) \mathrm{m}$ pec & $+1168^{c}$ & 16.35 & $<0.20^{b}$ & $<0.10$ & 15.6 \\
\hline ESO302-G014 & AM0349-383 & field & $\operatorname{Im}$ pec & +881 & 14.84 & $4.41^{d}$ & 0.56 & $\ldots$ \\
\hline ESO358-G060 & FCC $302^{e}$ & Fornax Cluster & $\mathrm{IB}(\mathrm{s}) \mathrm{m}:$ & +803 & 15.86 & $12.27^{f}$ & 3.97 & 20.0 \\
\hline IC 1613 & DDO 8, UGC 668 & Local Group & $\mathrm{IB}(\mathrm{s}) \mathrm{m}$ & -234 & 9.88 & $698^{g}$ & 0.89 & 0.73 \\
\hline IC 2032 & ESO156-G042, AM0405-552 & Dorado & $\mathrm{IAB}(\mathrm{s}) \mathrm{m}$ pec: & $+1066^{h}$ & 14.78 & $2.87^{h}$ & 0.35 & 17.2 \\
\hline IC 5152 & ESO237-G027, AM2159-513 & field & $\mathrm{IA}(\mathrm{s}) \mathrm{m}$ & +124 & 11.03 & $98.0^{i}$ & 0.35 & 2.07 \\
\hline NGC 2915 & ESO037-G003, AM0926-762 & field & $\mathrm{I} 0 ; \mathrm{BCD} ?$ & +468 & 12.93 & $145^{j}$ & 1.10 & 3.78 \\
\hline NGC 3109 & DDO 236, UGCA 194 & Antlia-Sextans ${ }^{k}$ & $\mathrm{SB}(\mathrm{s}) \mathrm{m}$ & +404 & 10.26 & $1110^{l}$ & 1.64 & 1.33 \\
\hline Sag DIG & ESO594-G004 & Local Group & $\mathrm{IB}(\mathrm{s}) \mathrm{m}$ & -77 & $13.99^{m}$ & $32.6^{n}$ & 1.21 & 1.11 \\
\hline
\end{tabular}

Table 2. Properties of Cassegrain Spectrograph employed at CTIO 1.5-m telescope. NOTE: ${ }^{a}$ Slit widths set for February and August 2001 runs, respectively.

\begin{tabular}{lc}
\hline \hline Property & Loral CCD \\
\hline Total area & 1200 pix $\times 800$ pix \\
Usable area & $1200 \mathrm{pix} \times 270$ pix \\
Pixel size & $15 \mu \mathrm{m}$ \\
Image scale & 1.3 arcsec pixel $^{-1}$ \\
Gain & $1.42 e^{-} \mathrm{ADU}^{-1}$ \\
Read-noise (rms) & $6.5 e^{-}$ \\
\hline \multicolumn{2}{c}{ Grating \#09 } \\
\hline Groove density & 300 lines mm $^{-1}$ \\
Blaze $\lambda(1$ st order) & $3560 \AA$ \\
Dispersion & $2.87 \AA$ pixel $^{-1}$ \\
Effective $\lambda$ range & $3500-6950 \AA$ \\
\hline \multicolumn{2}{c}{ Long slit } \\
\hline Length & 5.8 arcmin $^{\text {Width }}{ }^{a}$ \\
\hline
\end{tabular}

and snow prevented observations for the first two nights; the final two nights were clear with near-zero lunar illumination. The slit angle was kept constant in an east-west orientation for all observations. The central positions of the long slit for each target are listed in Table 3. After visual inspection of an image for each target, the centre of the long-slit was placed at the brightest candidate H II region for each galaxy.

Data reductions were carried out in the standard manner using IRAF ${ }^{1}$ routines. The raw two-dimensional images were subtracted for bias and trimmed. Dome flat exposures were used to remove pixel-to-pixel variations in response. Twilight flats were acquired at dusk each night to correct for variations over larger spatial scales. To correct for the "slit function" in the spatial direction, the variation of illumination along the slit was taken into account using dome and twilight flats. Multiple exposures of a given galaxy facilitated the removal of cosmic rays. Wavelength calibration was obtained using heliumargon (He-Ar) arc lamp exposures taken throughout each night.

${ }^{1}$ IRAF is distributed by the National Optical Astronomical Observatories, which is operated by the Associated Universities for Research in Astronomy, Inc., under contract to the National Science Foundation. 
Table 3. Log of observations. Column (1): Galaxy name in alphabetical order (within each association). Column (2): Date of observation. Columns (3) and (4): Central position of the long-slit: right ascension in hours, minutes and seconds, and declination in degrees, minutes, and seconds (Epoch J2000). Column (5): Number of exposures obtained and the length of each exposure in seconds. Column (6): Total exposure time. Column (7): Mean effective airmass. Column (8): [O III] $\lambda 4363$ : detected, upper limit, or not detected. Column (9): Relative root-meansquare error in the sensitivity function obtained from observations of standard stars. NOTES: ${ }^{a}$ H II region label from Webster et al. (1983). ${ }^{b}$ H II region number from Miller (1996). ${ }^{c}$ Only [O III] $\lambda 5007$ and $\mathrm{H} \alpha$ detected. ${ }^{d}$ H II region number from Hodge et al. (1990); also known as S3. ${ }^{e}$ H II region label from Talent (1980) and Webster et al. (1983). ${ }^{f}$ H II region number from Richer \& McCall (1992). ${ }^{g}$ H II region number from Skillman et al. (1989b) and Strobel et al. (1991).

\begin{tabular}{|c|c|c|c|c|c|c|c|c|}
\hline Galaxy & $\begin{array}{l}2001 \text { Date } \\
\text { (UT) }\end{array}$ & $\begin{array}{c}\alpha_{\text {slit }}(\mathrm{J} 2000) \\
\left({ }^{h m s}\right)\end{array}$ & $\begin{array}{c}\delta_{\text {slit }}(\mathrm{J} 2000) \\
\left({ }^{\circ}{ }^{\prime \prime \prime}\right)\end{array}$ & $N_{\text {exp }}$ & $\begin{array}{l}t_{\text {total }} \\
(\mathrm{s})\end{array}$ & $\langle X\rangle$ & {$[\mathrm{O} \mathrm{III}] \lambda 4363$} & $\begin{array}{l}\text { RMS } \\
\text { (mag) }\end{array}$ \\
\hline (1) & (2) & (3) & (4) & (5) & (6) & (7) & (8) & (9) \\
\hline \multicolumn{9}{|c|}{ Centaurus A group dwarfs } \\
\hline $\mathrm{A} 1243-335$ (H II\#A) $^{a}$ & $2 \mathrm{Feb}$ & 124602.4 & -335010.0 & $12 \times 600$ & 7200 & 1.18 & & $3.5 \%$ \\
\hline A1324-412 & $4 \mathrm{Feb}$ & 132737.2 & -412829.4 & $2 \times 1200$ & 2400 & 1.47 & & $6.5 \%$ \\
\hline A1334-277 & $4 \mathrm{Feb}$ & 133720.3 & -280215.8 & $8 \times 900$ & 7200 & 1.13 & $\ldots$ & $6.5 \%$ \\
\hline A1346-358 (H II\#A) ${ }^{a}$ & $3 \mathrm{Feb}$ & 134918.7 & $\begin{array}{lll}-36 & 03 & 10.4\end{array}$ & $8 \times 900$ & 7200 & 1.08 & detected & $4.1 \%$ \\
\hline DDO 161 & $3 \mathrm{Feb}$ & 130316.7 & -172503.2 & $7 \times 900$ & 6300 & 1.36 & upper limit & $4.1 \%$ \\
\hline NGC 5264 & $2 \mathrm{Feb}$ & 134137.0 & -295429.0 & $9 \times 600$ & 5400 & 1.05 & $\ldots$ & $3.5 \%$ \\
\hline \multicolumn{9}{|c|}{ Sculptor group dwarfs } \\
\hline AM0106-382 & 20 Aug & 010824.9 & -381159.8 & $1 \times 300,2 \times 1800$ & 3900 & 1.18 & $\ldots$ & $5.0 \%$ \\
\hline ESO 347-G017 & 19 Aug & 232656.3 & -372053.7 & $3 \times 1800$ & 5400 & 1.21 & upper limit & $5.0 \%$ \\
\hline ESO $348-$ G009 & 20 Aug & 234926.5 & -374542.1 & $1 \times 300,2 \times 1800$ & 3900 & 1.32 & & $5.0 \%$ \\
\hline UGCA $442(\mathrm{H} \mathrm{II \# 2})^{b}$ & 19 Aug & 234345.4 & -315727.4 & $1 \times 600,4 \times 1800$ & 7800 & 1.07 & upper limit & $5.0 \%$ \\
\hline \multicolumn{9}{|c|}{ Other southern dwarfs } \\
\hline $\mathrm{A} 0355-465{\text { (H II\#B })^{a}}^{a}$ & $3 \mathrm{Feb}$ & 035722.5 & -462200.2 & $6 \times 900$ & 5400 & 1.11 & upper limit & $4.1 \%$ \\
\hline ESO $302-\mathrm{G}_{0} 14^{c}$ & $2 \mathrm{Feb}$ & 035140.9 & -382709.5 & $1 \times 206,4 \times 3600$ & 3806 & 1.17 & $\ldots$ & $3.5 \%$ \\
\hline ESO $358-$ G060 & $4 \mathrm{Feb}$ & 034511.7 & -353422.9 & $7 \times 900$ & 6300 & 1.18 & upper limit & $6.5 \%$ \\
\hline IC $1613\left(\mathrm{H} \mathrm{II \# 13)^{d }}\right.$ & 20 Aug & 010447.3 & +020641.9 & $1 \times 300,3 \times 1800$ & 5700 & 1.19 & upper limit & $5.0 \%$ \\
\hline IC 1613 (H II\#37) $^{d}$ & 19 Aug & 010500.8 & +020426.1 & $1 \times 600,2 \times 1800$ & 4200 & 1.20 & detected & $5.0 \%$ \\
\hline IC 2032 & $2 \mathrm{Feb}$ & 040702.9 & -551931.2 & $12 \times 600$ & 7200 & 1.46 & $\ldots$ & $3.5 \%$ \\
\hline IC $5152(\mathrm{H} \mathrm{II \# A})^{e}$ & 20 Aug & 220251.8 & -511712.0 & $1 \times 300,3 \times 1800$ & 5700 & 1.43 & detected & $5.0 \%$ \\
\hline NGC 2915 & $3 \mathrm{Feb}$ & 092628.7 & -763731.2 & $6 \times 900$ & 5400 & 1.47 & $\ldots$ & $4.1 \%$ \\
\hline NGC 3109 (H II\#6) ${ }^{f}$ & $4 \mathrm{Feb}$ & 100304.2 & -260918.5 & $6 \times 900$ & 5400 & 1.07 & $\ldots$ & $6.5 \%$ \\
\hline Sag DIG $(\text { H II\#3 })^{g}$ & 19 Aug & 193002.3 & -174135.1 & $6 \times 1800$ & 10800 & 1.05 & $\cdots$ & $5.0 \%$ \\
\hline
\end{tabular}

Flux calibration was obtained using exposures of standard stars Feige 56, LTT 1788, LTT 3864 (February run); and LTT 1020, LTT 7379, LTT 9239 (August run). The flux accuracy is listed in Table 3. Final one-dimensional spectra for each H II region were obtained via unweighted summed extractions. For H II regions which were previously unidentified (i.e., other than those listed in Col. 1, Table 3), individual H II regions or aperture extractions are numbered along the slit increasing to the east. Representative H II region spectra are shown in Fig. 1; the spectrum for IC 1613 H II region \#37 is displayed in Fig. 2 to highlight faint emission lines.

\section{Measurements and analysis}

Emission-line strengths were measured using locallydeveloped software. Flux ratios were corrected for underlying Balmer absorption with an equivalent width $2 \AA$ (McCall et al. 1985). Corrections and analyses were performed with SNAP (Spreadsheet Nebular Analysis Package, Krawchuk et al. 1997). In the absence of [O III] $\lambda 4363$, the electron temperature was assumed to be $T_{e}=10^{4} \mathrm{~K}$ for computations. Where the density-dependent line ratio, $I([\mathrm{~S}$ II $] \lambda 6716) / I([\mathrm{~S}$ II $] \lambda 6731)$, could not be formed, a value of $n_{e}=100 \mathrm{~cm}^{-3}$ was adopted. Values of the reddening are derived using $\mathrm{H} \alpha$ and $\mathrm{H} \beta$ fluxes with the method described in Lee et al. (2003a). Errors in the reddening are computed from the maximum and minimum values of the reddening based upon $2 \sigma$ errors in fits to emission lines.
Observed flux $(F)$ and corrected intensity $(I)$ ratios are listed in Tables 4 to 7 inclusive. The listed errors for the observed flux ratios at each wavelength $\lambda$ account for the errors in the fits to the line profiles, their surrounding continua, and the relative error in the sensitivity function stated in Table 3 . Errors for observed ratios do not include the error in the flux at the $\mathrm{H} \beta$ reference line. The uncertainty in the correction for underlying Balmer absorption was assumed to be zero. Errors in the corrected intensity ratios account for maximum and minimum errors in the flux of the specified line and of the $\mathrm{H} \beta$ reference line; errors in the reddening are not included.

Derived properties are listed in Table 8 . The listed properties include $\mathrm{H} \beta$ intensities corrected for underlying Balmer absorption and reddening, derived and adopted values of the reddening, observed $\mathrm{H} \beta$ emission equivalent widths, and derived $\mathrm{O}^{+}$and $\mathrm{O}^{+2}$ electron temperatures (see next section). Despite the small number, the three [O III] $\lambda 4363$ detections are found in galaxies where $W_{e}(\mathrm{H} \beta) \gtrsim 40 \AA$ and $I(\mathrm{H} \beta) \gtrsim$ $7 \times 10^{-15} \mathrm{erg} \mathrm{s}^{-1} \mathrm{~cm}^{-2}$.

\section{Nebular abundances}

\subsection{Oxygen abundances: Direct method}

The direct or standard method of obtaining oxygen abundances from emission lines is applicable to any galaxy where [O III] $\lambda 4363$ is detectable and for which the doubly ionized $\mathrm{O}^{+2}$ ion is the dominant form of oxygen 
Table 4. Observed and corrected line ratios for Centaurus A group dwarf galaxies. Wavelengths are listed in $\AA$. For a given galaxy whose H II regions have not been previously identified (see Table 3), the number of the H II region aperture extraction increases to the east. $F$ is the observed flux ratio with respect to $\mathrm{H} \beta$. I is the corrected intensity ratio, corrected for underlying Balmer absorption and the adopted reddening listed in Table 8. The errors in the observed line ratios account for the errors in the fits to the line profiles, the surrounding continua, and the relative error in the sensitivity function listed in Table 3; flux errors in the $\mathrm{H} \beta$ reference line are not included. Errors in the corrected line ratios account for errors in the specified line and in the $\mathrm{H} \beta$ reference line.

\begin{tabular}{|c|c|c|c|c|c|c|}
\hline \multirow[b]{2}{*}{ Identification $(\AA)$} & \multicolumn{2}{|c|}{ A1243-335 H II\#A } & \multicolumn{2}{|c|}{ A1324-412 ap1 } & \multicolumn{2}{|c|}{ A1334-277 ap1 } \\
\hline & $F$ & $I$ & $F$ & $I$ & $F$ & $I$ \\
\hline [O II] 3727 & $107 \pm 15$ & $117 \pm 31$ & $\ldots$ & $\ldots$ & $105 \pm 15$ & $94 \pm 31$ \\
\hline $\mathrm{H} \beta 4861$ & $100 \pm 10$ & $100 \pm 12$ & $100 \pm 15$ & $100 \pm 19$ & $100 \pm 13$ & $100 \pm 17$ \\
\hline [O III] 4959 & $163.0 \pm 7.1$ & $145 \pm 26$ & $\ldots$ & $\ldots$ & $70 \pm 12$ & $63 \pm 22$ \\
\hline [O III] 5007 & $456.6 \pm 8.5$ & $405 \pm 65$ & $\ldots$ & $\ldots$ & $176 \pm 14$ & $158 \pm 43$ \\
\hline $\mathrm{H} \alpha 6563$ & $357.4 \pm 7.5$ & $286 \pm 47$ & $366 \pm 20$ & $286 \pm 80$ & $291 \pm 12$ & $268 \pm 66$ \\
\hline \multirow{2}{*}{ [N II] 6583} & $4.7 \pm 6.1$ & $3.7 \pm 5.3$ & $\ldots$ & $\ldots$ & $12 \pm 10$ & $10 \pm 11$ \\
\hline & \multicolumn{2}{|c|}{ A1346-358 H II\#A } & \multicolumn{2}{|c|}{ DDO 161 ap1 } & \multicolumn{2}{|c|}{ NGC 5264 ap1 } \\
\hline Identification $(\AA)$ & $F$ & $I$ & $F$ & $I$ & $F$ & $I$ \\
\hline [O II] 3727 & $235.5 \pm 1.9$ & $415 \pm 36$ & $190.5 \pm 3.6$ & $315 \pm 29$ & $377 \pm 20$ & $390 \pm 61$ \\
\hline He II 3834 + H9 & $5.3 \pm 1.0$ & $13.9 \pm 4.5$ & $\ldots$ & $\ldots$ & $\ldots$ & $\ldots$ \\
\hline [Ne III] 3869 & $19.8 \pm 1.2$ & $31.7 \pm 3.7$ & $\ldots$ & $\ldots$ & $\ldots$ & $\ldots$ \\
\hline He I 3889 + H8 & $18.3 \pm 1.2$ & $33.7 \pm 4.4$ & $\ldots$ & $\ldots$ & $\ldots$ & $\ldots$ \\
\hline$[\mathrm{Ne}$ III] $3967+\mathrm{H} \epsilon$ & $19.05 \pm 0.77$ & $32.8 \pm 3.5$ & $\ldots$ & $\ldots$ & $\ldots$ & $\ldots$ \\
\hline $\mathrm{H} \delta 4101$ & $20.29 \pm 0.79$ & $31.7 \pm 3.3$ & $14.4 \pm 1.9$ & $26.7 \pm 6.2$ & $\ldots$ & $\ldots$ \\
\hline $\mathrm{H} \gamma 4340$ & $47.14 \pm 0.75$ & $60.6 \pm 5.4$ & $46.4 \pm 2.4$ & $61.4 \pm 7.3$ & $27.3 \pm 8.3$ & $43 \pm 25$ \\
\hline [O III] 4363 & $1.93 \pm 0.59$ & $2.34 \pm 0.82$ & $<6.6$ & $<7.7$ & $\ldots$ & $\ldots$ \\
\hline He I 4472 & $3.52 \pm 0.53$ & $4.04 \pm 0.81$ & $\ldots$ & $\ldots$ & $\ldots$ & $\ldots$ \\
\hline $\mathrm{H} \beta 4861$ & $100.0 \pm 1.4$ & $100.0 \pm 4.4$ & $100.0 \pm 2.1$ & $100.0 \pm 4.8$ & $100.0 \pm 7.2$ & $100.0 \pm 9.3$ \\
\hline [O III] 4959 & $95.6 \pm 2.3$ & $90.6 \pm 8.3$ & $92.8 \pm 2.3$ & $85.9 \pm 8.2$ & $34.4 \pm 5.7$ & $28.8 \pm 7.6$ \\
\hline [O III] 5007 & $292.8 \pm 2.8$ & $273 \pm 24$ & $278.9 \pm 2.8$ & $254 \pm 23$ & $83.4 \pm 7.1$ & $69 \pm 13$ \\
\hline He I 5876 & $15.46 \pm 0.50$ & $11.4 \pm 1.1$ & $17.0 \pm 1.9$ & $12.4 \pm 2.1$ & $\ldots$ & $\ldots$ \\
\hline [N II] 6548 & $15.3 \pm 2.7$ & $9.9 \pm 2.2$ & $\ldots$ & $\ldots$ & $20.4 \pm 7.6$ & $15.0 \pm 7.1$ \\
\hline $\mathrm{H} \alpha 6563$ & $443.0 \pm 3.4$ & $286 \pm 25$ & $440.2 \pm 3.0$ & $286 \pm 26$ & $374.6 \pm 9.5$ & $286 \pm 39$ \\
\hline [N II] 6583 & $39.6 \pm 2.7$ & $25.4 \pm 3.2$ & $8.9 \pm 2.5$ & $5.7 \pm 1.9$ & $63.6 \pm 7.8$ & $47 \pm 10$ \\
\hline He I 6678 & $4.04 \pm 0.68$ & $2.55 \pm 0.56$ & $\ldots$ & $\ldots$ & $\ldots$ & $\ldots$ \\
\hline [S II] 6716 & $39.45 \pm 0.80$ & $24.8 \pm 2.2$ & $22.0 \pm 2.0$ & $13.9 \pm 2.0$ & $93.4 \pm 7.7$ & $68 \pm 12$ \\
\hline [S II] 6731 & $27.45 \pm 0.75$ & $17.2 \pm 1.4$ & $15.8 \pm 1.9$ & $10.0 \pm 1.7$ & $60.5 \pm 7.2$ & $44.0 \pm 9.6$ \\
\hline
\end{tabular}

(Osterbrock 1989). A summary of the "standard" method by which oxygen abundances are derived can be found in Dinerstein (1990). Computations were performed with SNAP. The relative abundances of singly- and doubly-ionized oxygen and the total oxygen abundance by number are computed using the method described by Lee et al. (2003a). An $\mathrm{O}^{+2} / \mathrm{H}$ abundance was computed using an $\mathrm{O}^{+2}$ temperature, derived from the intensity of the [O III] $\lambda 4363$ and [O III] $\lambda \lambda 4959,5007$ lines, and an $\mathrm{O}^{+} / \mathrm{H}$ abundance was computed using an $\mathrm{O}^{+}$temperature derived using Eq. (2) from Izotov et al. (1997b).

Direct ([O III $]$ 24363) abundances were obtained for three galaxies (A1346-358, IC 1613 H II\#37, and IC 5152 H II\#A) and are listed in Table 9. Errors in direct oxygen abundances were computed from the maximum and minimum possible values, given the errors in the line intensities; errors in reddening and temperature are not included. For the remaining galaxies, secondary techniques using the bright emission lines of ionized oxygen are utilized to derive oxygen abundances.

\subsection{Oxygen abundances: Bright-line method}

In the absence of [O III] $\lambda 4363$, the bright-line or empirical method was used to compute oxygen abundances. The method is so called because the oxygen abundance is given in terms of the bright [O II] and [O III] lines. Pagel et al. (1979) suggested that the ratio

$$
R_{23}=\frac{I([\mathrm{O} \mathrm{II}] \lambda 3727)+I([\mathrm{O} \mathrm{III}] \lambda \lambda 4959,5007)}{I(\mathrm{H} \beta)}
$$

could be used as an abundance indicator. For the dwarf galaxies in the present work, the calibrations by McGaugh $(1991,1994)$ and Pilyugin (2000) were used to derive oxygen abundances.

McGaugh $(1991,1994)$ produced a set of photoionization models using $R_{23}$ and

$$
O_{32}=\frac{I([\mathrm{O} \mathrm{III}] \lambda \lambda 4959,5007)}{I([\mathrm{O} \mathrm{II}] \lambda 3727)}
$$

to estimate the oxygen abundance. However, $R_{23}$ is not a monotonic function of the oxygen abundance. At a given value of $R_{23}$, there are two possible choices of the oxygen abundance as shown in Fig. 3. Each filled circle represents an $\mathrm{HII}$ region from dIs in the control sample with measured [O III] ג4363. Model curves from McGaugh (1991) are superimposed. A long-dashed line marks the approximate boundary below (above) which the lower branch (upper branch) occurs. Despite the fact the oxygen abundances for H II regions in the control sample of dIs range from about one-tenth to about one-half of the solar value, these H II regions are clustered around the "knee" of the curves, where ambiguity is greatest 
Table 5. Observed and corrected line ratios for Sculptor group dwarf galaxies; same comments as Table 4. NOTE: ${ }^{a}$ The recession velocity of emission lines is $v_{\odot} / c \simeq+0.11$, placing this galaxy in the background. No corrections are applied.

\begin{tabular}{|c|c|c|c|c|c|c|}
\hline \multirow[b]{2}{*}{ Identification $(\AA)$} & \multicolumn{2}{|c|}{ AM0106-382 ap1 } & \multicolumn{2}{|c|}{ AM0106-382 ар2 } & \multicolumn{2}{|c|}{ AM0106-382 ар3 } \\
\hline & $F$ & $I$ & $F$ & $I$ & $F$ & $I$ \\
\hline [O II] 3727 & $168 \pm 21$ & $155 \pm 40$ & $165 \pm 15$ & $149 \pm 29$ & $178 \pm 17$ & $158 \pm 31$ \\
\hline $\mathrm{H} \beta 4861$ & $100.0 \pm 9.9$ & $100.0 \pm 12$ & $100.0 \pm 6.4$ & $100.0 \pm 8.9$ & $100.0 \pm 6.4$ & $100.0 \pm 8.9$ \\
\hline [O III] 4959 & $22.3 \pm 7.7$ & $20.6 \pm 9.8$ & $37.6 \pm 4.7$ & $33.9 \pm 7.6$ & $45.8 \pm 5.7$ & $40.6 \pm 9.1$ \\
\hline [O III] 5007 & $62.4 \pm 7.9$ & $58 \pm 15$ & $124.8 \pm 5.8$ & $113 \pm 18$ & $162.1 \pm 7.1$ & $144 \pm 22$ \\
\hline [N II] 6548 & $\ldots$ & $\ldots$ & $1.3 \pm 4.2$ & $1.1 \pm 3.9$ & $2.8 \pm 3.9$ & $2.5 \pm 3.7$ \\
\hline $\mathrm{H} \alpha 6563$ & $239.7 \pm 8.9$ & $225 \pm 42$ & $266.2 \pm 5.3$ & $245 \pm 35$ & $287.4 \pm 4.9$ & $260 \pm 37$ \\
\hline [N II] 6583 & $12.4 \pm 7.2$ & $11.4 \pm 8.1$ & $7.0 \pm 4.3$ & $6.3 \pm 4.4$ & $5.02 \pm 3.9$ & $4.4 \pm 3.9$ \\
\hline [S II] 6716 & $39.8 \pm 8.0$ & $37 \pm 12$ & $29.1 \pm 5.2$ & $26.3 \pm 7.2$ & $23.3 \pm 4.1$ & $20.6 \pm 5.6$ \\
\hline \multirow[t]{2}{*}{ [S II] 6731} & $20.7 \pm 7.0$ & $19.1 \pm 8.9$ & $15.2 \pm 4.4$ & $13.7 \pm 5.2$ & $15.7 \pm 3.3$ & $13.9 \pm 4.3$ \\
\hline & \multicolumn{2}{|c|}{ ESO 347-G017 ap1 } & \multicolumn{2}{|c|}{ ESO 347-G017 ap2 } & \multicolumn{2}{|c|}{ ESO 347-G017 ap3 } \\
\hline Identification $(\AA)$ & $F$ & $I$ & $F$ & $I$ & $F$ & $I$ \\
\hline [O II] 3727 & $215.3 \pm 3.4$ & $200 \pm 22$ & $159.7 \pm 4.2$ & $171 \pm 21$ & $244 \pm 21$ & $296 \pm 69$ \\
\hline [Ne III] 3869 & $39.0 \pm 4.3$ & $36.1 \pm 6.5$ & $44.2 \pm 6.4$ & $45.5 \pm 9.9$ & $\ldots$ & $\ldots$ \\
\hline $\mathrm{H} \gamma 4340$ & $33.5 \pm 1.9$ & $42.7 \pm 6.5$ & $28.0 \pm 3.1$ & $41.7 \pm 9.3$ & $\ldots$ & $\ldots$ \\
\hline [O III] 4363 & $<4.9(2 \sigma)$ & $<4.4(2 \sigma)$ & $<4.2(2 \sigma)$ & $<4.4(2 \sigma)$ & $\ldots$ & $\ldots$ \\
\hline $\mathrm{H} \beta 4861$ & $100.0 \pm 2.3$ & $100.0 \pm 5.8$ & $100.0 \pm 3.5$ & $100.0 \pm 6.5$ & $100 \pm 10$ & $100 \pm 13$ \\
\hline [O III] 4959 & $109.2 \pm 3.2$ & $98 \pm 11$ & $136.0 \pm 3.1$ & $118 \pm 14$ & $72.6 \pm 6.8$ & $62 \pm 14$ \\
\hline [O III] 5007 & $312.4 \pm 3.9$ & $281 \pm 31$ & $414.0 \pm 3.8$ & $359 \pm 42$ & $192.5 \pm 8.4$ & $160 \pm 31$ \\
\hline He I 5876 & $17.2 \pm 2.7$ & $15.4 \pm 3.4$ & $20.3 \pm 2.4$ & $16.3 \pm 3.1$ & $\ldots$ & $\ldots$ \\
\hline [N II] 6548 & $8.7 \pm 2.8$ & $7.7 \pm 2.9$ & $7.0 \pm 2.7$ & $5.6 \pm 2.5$ & $\ldots$ & $\ldots$ \\
\hline $\mathrm{H} \alpha 6563$ & $315.9 \pm 3.5$ & $286 \pm 31$ & $363.6 \pm 3.4$ & $286 \pm 33$ & $418 \pm 12$ & $286 \pm 55$ \\
\hline [N II] 6583 & $11.8 \pm 2.9$ & $10.5 \pm 3.2$ & $12.2 \pm 2.8$ & $9.6 \pm 2.8$ & $10.8 \pm 9.8$ & $5.8 \pm 7.7$ \\
\hline [S II] 6716 & $42.0 \pm 1.8$ & $37.0 \pm 4.6$ & $24.6 \pm 2.0$ & $18.7 \pm 3.0$ & $41.9 \pm 7.9$ & $27.8 \pm 9.1$ \\
\hline \multirow[t]{2}{*}{ [S II] 6731} & $25.3 \pm 1.6$ & $22.3 \pm 3.1$ & $17.3 \pm 1.9$ & $13.1 \pm 2.4$ & $20.7 \pm 6.3$ & $13.7 \pm 6.1$ \\
\hline & \multicolumn{2}{|c|}{ ESO 348-G009 ap1 } & \multicolumn{2}{|c|}{ ESO 348-G009 ap2 ${ }^{a}$} & \multicolumn{2}{|c|}{ UGCA 442 H II\#2 } \\
\hline Identification $(\AA)$ & $F$ & $I$ & $F$ & $I$ & $F$ & $I$ \\
\hline [O II] 3727 & $317 \pm 29$ & $283 \pm 72$ & $589 \pm 54$ & $\ldots$ & $221.4 \pm 4.9$ & $237 \pm 27$ \\
\hline $\mathrm{H} \gamma 4340$ & $\ldots$ & $\ldots$ & $\ldots$ & $\ldots$ & $36.2 \pm 2.4$ & $47.3 \pm 7.6$ \\
\hline [O III] 4363 & $\ldots$ & $\ldots$ & $\ldots$ & $\ldots$ & $<3.8(2 \sigma)$ & $<3.6(2 \sigma)$ \\
\hline $\mathrm{H} \beta 4861$ & $100 \pm 12$ & $100 \pm 15$ & $100 \pm 33$ & $\ldots$ & $100.0 \pm 2.5$ & $100.0 \pm 5.9$ \\
\hline [O III] 4959 & $53 \pm 10$ & $47 \pm 16$ & $101 \pm 32$ & $\ldots$ & $53.6 \pm 2.2$ & $47.5 \pm 5.9$ \\
\hline [O III] 5007 & $118 \pm 12$ & $106 \pm 28$ & $583 \pm 41$ & $\ldots$ & $155.0 \pm 2.7$ & $137 \pm 15$ \\
\hline He I 5876 & $\ldots$ & $\ldots$ & $\ldots$ & $\ldots$ & $16.5 \pm 2.8$ & $13.5 \pm 3.2$ \\
\hline [N II] 6548 & $\ldots$ & $\ldots$ & $\ldots$ & $\ldots$ & $6.3 \pm 2.3$ & $4.9 \pm 2.2$ \\
\hline $\mathrm{H} \alpha 6563$ & $254.1 \pm 9.1$ & $233 \pm 49$ & & $\ldots$ & $356.2 \pm 2.9$ & $286 \pm 32$ \\
\hline [N II] 6583 & $10.7 \pm 7.3$ & $9.6 \pm 8.0$ & $\ldots$ & $\ldots$ & $14.3 \pm 2.4$ & $11.3 \pm 2.6$ \\
\hline [S II] 6716 & $44.2 \pm 7.7$ & $39 \pm 13$ & $\ldots$ & $\ldots$ & $36.8 \pm 1.5$ & $28.7 \pm 3.6$ \\
\hline [S II] 6731 & $20.6 \pm 5.9$ & $18.4 \pm 8.1$ & 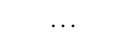 & $\ldots$ & $22.7 \pm 1.4$ & $17.7 \pm 2.5$ \\
\hline
\end{tabular}

about the choice of the appropriate branch in the absence of [O III] $] 4363$.

Fortunately, $I([\mathrm{~N} \mathrm{II}] \lambda 6583) / I([\mathrm{O}$ II $] \lambda 3727)$, or the $[\mathrm{N} \mathrm{II}] /[\mathrm{O}$ II] intensity ratio can discriminate between the lower and upper branches (McCall et al. 1985; McGaugh 1991, 1994). The strength of the [N II] $\lambda 6583$ line is roughly proportional to the nitrogen abundance and the $[\mathrm{N} \mathrm{II}] /[\mathrm{O} \mathrm{II}]$ intensity ratio is relatively insensitive to ionization. McGaugh (1994) has shown that in galaxies ranging from sub-solar to solar metallicities, [N II]/[O II] can vary by one to two orders of magnitude and that $[\mathrm{N} \mathrm{II}] /[\mathrm{O} \mathrm{II}]$ is roughly below (above) 0.1 at low (high) oxygen abundance. A plot of the $[\mathrm{N} \mathrm{II}] /[\mathrm{O} \mathrm{II}]$ intensity ratio versus $R_{23}$ is shown in Fig. 4 . While most H II regions in the present sample lie in the lower branch regime, they lie to the left of the locus of points defined by H II regions in nearby dIs. This is expected for metal-poor galaxies as ionization effects become more prominent (McGaugh 1991). The corrected [N II]/[O II] was used to determine the branch for computing the oxygen abundance in both the McGaugh and Pilyugin calibrations.

For the McGaugh (1997, private communication) calibration, analytical equations for the oxygen abundance are given in terms of $x \equiv \log R_{23}$ and $y \equiv \log O_{32}$. The expressions for lower branch and upper branch oxygen abundances are

$$
\begin{aligned}
12+\log (\mathrm{O} / \mathrm{H})_{\text {lower }}= & 12-4.93+4.25 x-3.35 \sin (x) \\
& -0.26 y-0.12 \sin (y), \\
12+\log (\mathrm{O} / \mathrm{H})_{\text {upper }}= & 12-2.65-0.91 x+0.12 y \sin (x),
\end{aligned}
$$

respectively, where the argument of the trigonometric function is in radians.

Pilyugin suggested a new calibration for the bright-line method. His method at low metallicities accounts for the systematic uncertainties in the $R_{23}$ method, whereas at high metallicities, he obtains a relation for the oxygen abundance as a function of the intensities of the bright [O II] and [O III] lines. 


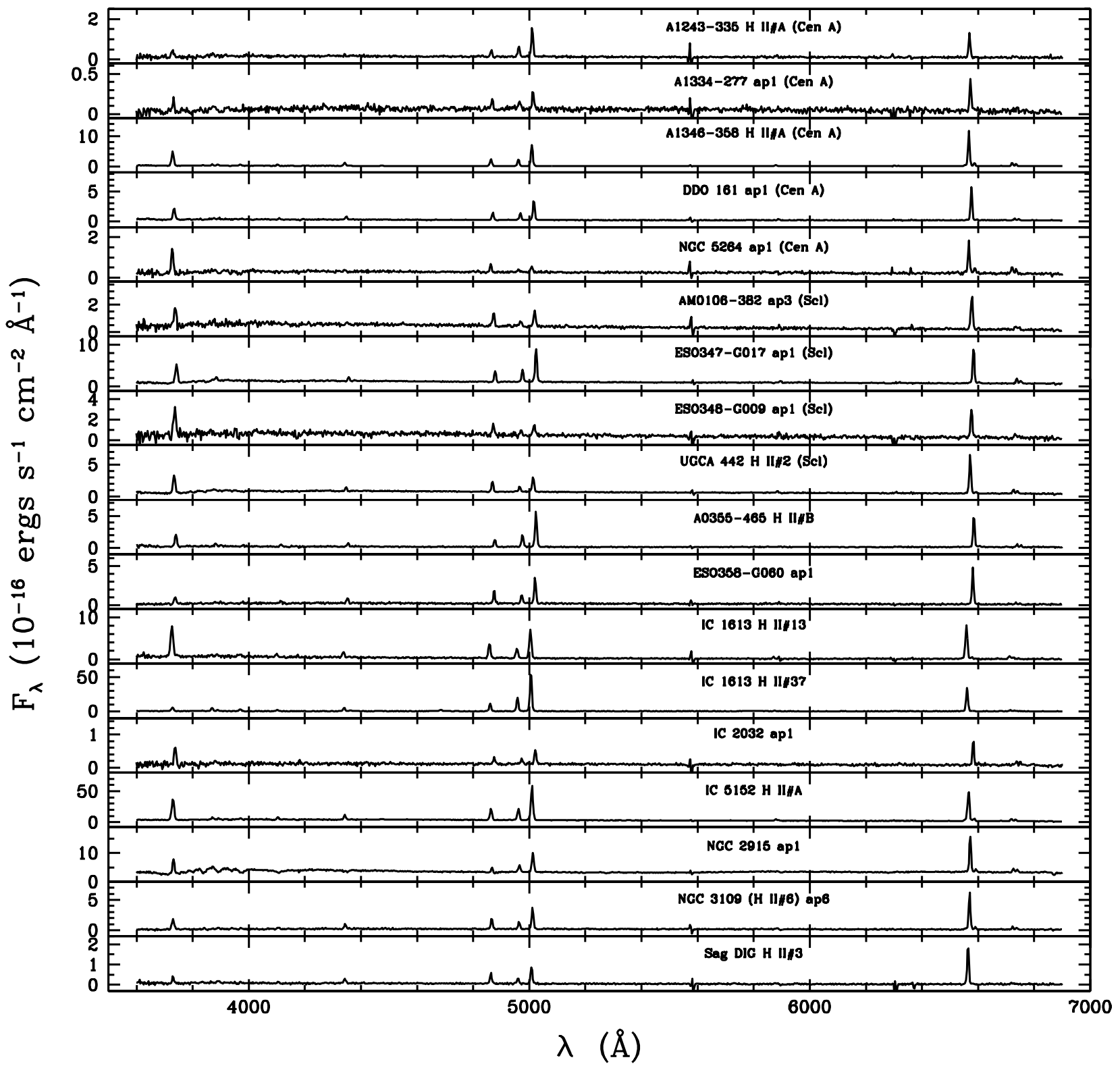

Fig. 1. Long-slit spectra. The flux per unit wavelength is plotted against wavelength in each panel.

For lower branch and upper branch abundances, we use Eq. (4) from Pilyugin (2000), and Eq. (8) from Pilyugin (2001a), respectively.

Bright-line oxygen abundances are derived and listed in Table 9. Figure 5 shows how the different determinations of the oxygen abundance vary with $O_{32}$ and $R_{23}$. Differences in derived oxygen abundance between the direct ([O III] 24363 ) and bright-line McGaugh methods, between the direct and brightline Pilyugin methods, and between the two bright-line methods are shown. The separations among the three methods appear to increase with increasing $O_{32}$. The difference between the McGaugh and Pilyugin calibrations (indicated by asterisks) appears to correlate with $\log O_{32}$; this effect is also observed by Skillman et al. (2003). IC 1613 H II\#37 with the largest measured $O_{32}\left(\log O_{32}=0.998\right)$ exhibits the largest overall discrepancy among the direct, McGaugh, and Pilyugin methods $(12+\log (\mathrm{O} / \mathrm{H})=7.62,7.88$, and 7.71, respectively $)$.

\subsection{Nitrogen-to-oxygen and neon-to-oxygen abundance ratios}

Based upon observations of $\mathrm{HII}$ regions in spiral and dwarf galaxies, nitrogen appears to be both a primary and secondary product of nucleosynthesis. It remains uncertain, however, whether nitrogen is produced mostly from short-lived massive stars or from longer-lived intermediate-mass stars. An extensive review of the possible origins for nitrogen is discussed by Henry et al. (2000).

Measurements of the nitrogen-to-oxygen ratio, $\mathrm{N} / \mathrm{O}$, have been used to differentiate between the different origins for nitrogen. It has been suggested that N/O can be used as a "clock" to measure the time since the last burst of star formation (e.g., Garnett 1990; Skillman et al. 1997, 2003). This scenario works if bursts of star formation are separated by long quiescent periods, if the delivery of nitrogen into the interstellar gas is 


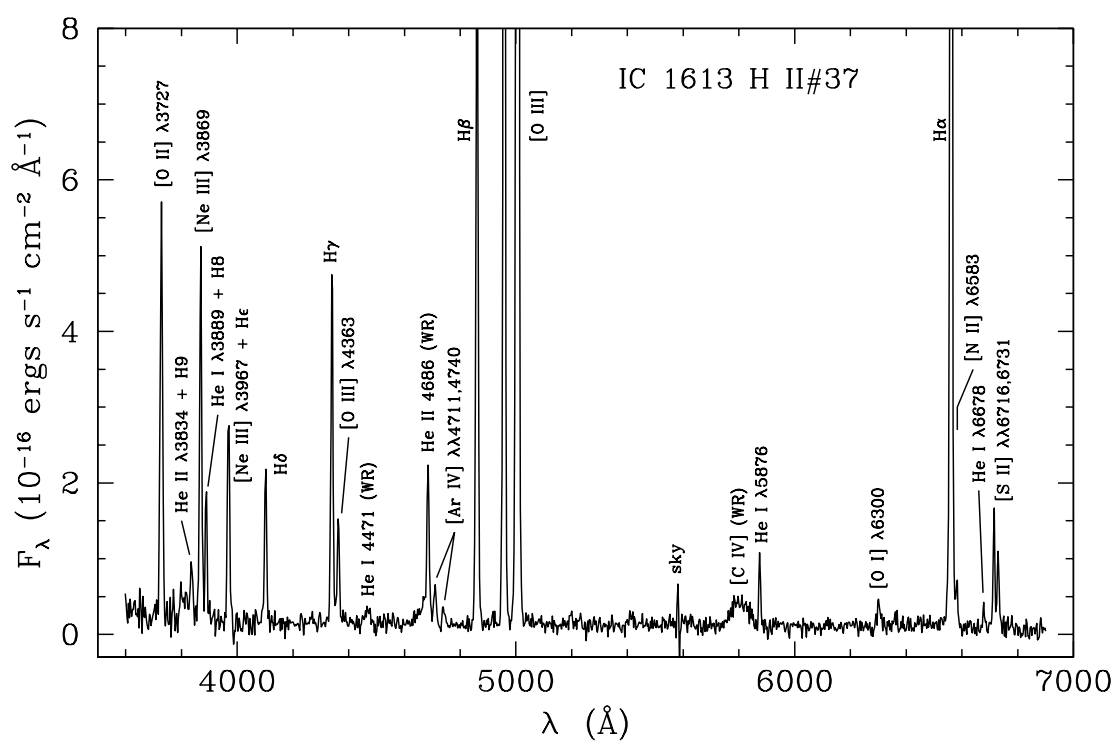

Fig. 2. IC 1613 H II\#37: The spectrum is redisplayed to highlight faint emission lines and broad Wolf-Rayet (W-R) features. The forbidden line [Ar IV] $\lambda 4711$ is likely blended with He I $\lambda 4713$.

delayed relative to oxygen, and if there is no significant metals loss. The result is that $\mathrm{N} / \mathrm{O}$ values are low at a given $\mathrm{O} / \mathrm{H}$ if a burst of star formation has occurred recently, whereas N/O values are high after a long quiescent period.

For low-abundance H II regions, $\log (\mathrm{N} / \mathrm{O}) \approx \log \left(\mathrm{N}^{+} / \mathrm{O}^{+}\right)$ is a good approximation (e.g., Garnett 1990). Assuming that $T_{e}\left(\mathrm{~N}^{+}\right)=T_{e}\left(\mathrm{O}^{+}\right)$, the nitrogen-to-oxygen abundance ratio is

$\mathrm{N} / \mathrm{O}=\frac{I([\mathrm{~N} \mathrm{II}] \lambda 6583)}{I([\mathrm{O} \mathrm{II}] \lambda 3727)} \frac{j\left([\mathrm{O} \mathrm{II}] \lambda 3727 ; n_{e}, T_{e}\left(\mathrm{O}^{+}\right)\right)}{j\left([\mathrm{~N} \mathrm{II}] \lambda 6583 ; n_{e}, T_{e}\left(\mathrm{O}^{+}\right)\right)}$,

where $j$ is the volume emissivity of the given line at density $n_{e}$ and temperature $T_{e}$. In the absence of [O III] 14363 , a brightline oxygen abundance was derived using the McGaugh calibration and an $\mathrm{O}^{+2}$ temperature was obtained using the correlation between oxygen abundance and temperature in Fig. 7 of McGaugh (1991). Subsequently, an $\mathrm{O}^{+}$temperature was estimated using the data in Table 1 of Vila-Costas \& Edmunds (1993). The nitrogen-to-oxygen abundance ratio was then computed by using the appropriate line intensities with Eq. (9) from Pagel et al. (1992).

Neon is a product of $\alpha$-processes in nucleosynthesis occurring in the same massive stars which produce oxygen. As a result, the neon-to-oxygen ratio, $\mathrm{Ne} / \mathrm{O}$, is expected to be constant with oxygen abundance. Assuming that doubly-ionized neon is found in the same zone as doubly-ionized oxygen and that $T_{e}\left(\mathrm{Ne}^{+2}\right)=T_{e}\left(\mathrm{O}^{+2}\right)$ and that $\log (\mathrm{Ne} / \mathrm{O}) \simeq \log \left(\mathrm{Ne}^{+2} / \mathrm{O}^{+2}\right)$, the neon-to-oxygen abundance ratio is

$\mathrm{Ne} / \mathrm{O}=\frac{I([\mathrm{Ne} \text { III }] \lambda 3869)}{I([\mathrm{O} \text { III }] \lambda 5007)} \frac{j\left([\mathrm{O} \text { III }] \lambda 5007 ; n_{e}, T_{e}\left(\mathrm{O}^{+2}\right)\right)}{j\left([\mathrm{Ne} \text { III }] \lambda 3869 ; n_{e}, T_{e}\left(\mathrm{Ne}^{+2}\right)\right)}$

In the absence of [O III] $\lambda 4363$, a bright-line temperature for the $\mathrm{O}^{+2}$ zone is obtained (see previous paragraph) and the neon-tooxygen abundance ratio is computed using the appropriate line intensities with Eq. (10) from Pagel et al. (1992).

Table 9 lists $\mathrm{N} / \mathrm{O}$ and $\mathrm{Ne} / \mathrm{O}$ abundance ratios. For the three galaxies with [O III] $\lambda 4363$ detections, $\mathrm{N} / \mathrm{O}$ and $\mathrm{Ne} / \mathrm{O}$ values derived using the direct method generally agree with those derived with the bright-line method, except that the direct value of $\log (\mathrm{N} / \mathrm{O})$ for IC $1613 \mathrm{H} \mathrm{II \# 37} \mathrm{is} 0.2$ dex larger than the brightline value. A few of the $\mathrm{Ne} / \mathrm{O}$ values are higher than those derived for blue compact dwarf galaxies: $\log (\mathrm{Ne} / \mathrm{O}) \approx-0.7$ (Izotov \& Thuan 1999), but [Ne III] $\lambda 3869$ flux measurements for galaxies in the present sample may be overestimated from noisy spectra.

\section{Discussion}

For bright-line determinations of oxygen abundances, the reported values for each galaxy are means of values derived from the McGaugh and the Pilyugin calibrations. New oxygen abundances are reported for eight galaxies from the southern sample: in the Cen A group, A1334-277, DDO 161, and NGC 5264; in the Scl group, AM0106-382, ESO347-G017 (but see Appendix A), and ESO348-G009; and finally, ESO358-G060, IC 2032, and NGC 2915.

\subsection{Comments on individual galaxies}

All of the galaxies in the southern sample are presented below in alphabetical order.

\subsubsection{A0355-465 (ESO249-G032)}

Webster et al. (1983) obtained spectra of two H II regions (\#A and \#B). Unfortunately, their [O III] $\lambda 4363$ measurement in $\mathrm{HII}$ region $\# \mathrm{~B}$ is very uncertain with their quoted error in excess of $40 \%$; they derived $12+\log (\mathrm{O} / \mathrm{H})=8.41$ and $\log (\mathrm{N} / \mathrm{O})=-1.70$. From our spectrum of $\mathrm{HII}$ region \#B, we obtain $12+\log (\mathrm{O} / \mathrm{H})=8.12$ and $\log (\mathrm{N} / \mathrm{O})=-1.61$ using the bright-line method. While our $I([\mathrm{O}$ III $] \lambda \lambda 4959,5007) / I(\mathrm{H} \beta)$ ratio is about $80 \%$ of that reported by Webster et al. (1983), our $I([\mathrm{O} \mathrm{II}] \lambda 3727) / I(\mathrm{H} \beta)$ ratio is about a factor of two lower. 
Table 6. Observed and corrected line ratios for other southern dwarf galaxies; same comments as Table 4. ESO302-G014 \#1 is not included here, as only [O III] $\lambda 5007$ and $\mathrm{H} \alpha$ were detected; the measured fluxes in ergs s $\mathrm{s} \mathrm{cm}^{-2}$ are $(2.13 \pm 0.44) \times 10^{-15}$ and $(2.73 \pm 0.43) \times 10^{-15}$, respectively. NOTE: ${ }^{a}$ A broad Wolf-Rayet feature was detected near He I $\lambda 4472$.

\begin{tabular}{|c|c|c|c|c|c|c|}
\hline \multirow[b]{2}{*}{ Identification $(\AA)$} & \multicolumn{2}{|c|}{ A0355-465 H II\#B } & \multicolumn{2}{|c|}{ ESO358-G060 ap1 } & \multicolumn{2}{|c|}{ IC 1613 H II\#13 } \\
\hline & $F$ & $I$ & $F$ & $I$ & $F$ & $I$ \\
\hline [O II] 3727 & $185.9 \pm 5.3$ & $277 \pm 28$ & $66.1 \pm 4.9$ & $64 \pm 12$ & $256.7 \pm 6.1$ & $251 \pm 28$ \\
\hline [Ne III] 3869 & $41.8 \pm 7.0$ & $58 \pm 13$ & $7.2 \pm 2.1$ & $7.0 \pm 2.6$ & $\ldots$ & $\ldots$ \\
\hline He I 3889 + H8 & $16.7 \pm 5.6$ & $31 \pm 16$ & $8.5 \pm 2.7$ & $11.5 \pm 6.0$ & $\ldots$ & $\ldots$ \\
\hline [Ne III] $3967+\mathrm{H} \epsilon$ & $19.1 \pm 4.9$ & $32 \pm 12$ & $9.7 \pm 2.7$ & $12.8 \pm 6.0$ & $19.08 \pm 4.1$ & $22.9 \pm 7.5$ \\
\hline $\mathrm{H} \delta 4101$ & $29.8 \pm 4.5$ & $43 \pm 10$ & $20.5 \pm 3.8$ & $23.2 \pm 7.2$ & $20.9 \pm 3.4$ & $24.0 \pm 6.1$ \\
\hline $\mathrm{H} \gamma 4340$ & $45.5 \pm 4.5$ & $57.1 \pm 9.6$ & $42.8 \pm 3.4$ & $45.0 \pm 8.7$ & $38.8 \pm 3.1$ & $41.1 \pm 6.5$ \\
\hline [O III] 4363 & $<6.4(2 \sigma)$ & $<7.1(2 \sigma)$ & $<5.8(2 \sigma)$ & $<5.6(2 \sigma)$ & $<7.3(2 \sigma)$ & $<7.1(2 \sigma)$ \\
\hline $\mathrm{H} \beta 4861$ & $100.0 \pm 2.8$ & $100.0 \pm 5.1$ & $100.0 \pm 4.1$ & $100.0 \pm 8.1$ & $100.0 \pm 2.3$ & $100.0 \pm 5.7$ \\
\hline [O III] 4959 & $166.8 \pm 5.2$ & $156 \pm 16$ & $68.7 \pm 2.8$ & $66 \pm 11$ & $67.1 \pm 1.6$ & $65.6 \pm 7.3$ \\
\hline [O III] 5007 & $486.1 \pm 6.4$ & $450 \pm 43$ & $202.4 \pm 3.4$ & $196 \pm 29$ & $204.4 \pm 1.9$ & $200 \pm 22$ \\
\hline He I 5876 & $\ldots$ & $\ldots$ & $\ldots$ & $\ldots$ & $11.4 \pm 4.3$ & $11.2 \pm 4.9$ \\
\hline [N II] 6548 & $\ldots$ & $\ldots$ & $\ldots$ & $\ldots$ & $7.3 \pm 1.7$ & $7.2 \pm 2.1$ \\
\hline $\mathrm{H} \alpha 6563$ & $403.6 \pm 3.7$ & $286 \pm 27$ & $258.2 \pm 3.2$ & $251 \pm 37$ & $227.5 \pm 2.1$ & $223 \pm 24$ \\
\hline [N II] 6583 & $15.6 \pm 3.0$ & $10.9 \pm 2.7$ & $4.5 \pm 2.6$ & $4.4 \pm 2.9$ & $10.9 \pm 1.7$ & $10.7 \pm 2.4$ \\
\hline [S II] 6716 & $32.6 \pm 3.5$ & $22.5 \pm 3.8$ & $12.3 \pm 2.1$ & $11.9 \pm 3.2$ & $19.7 \pm 1.8$ & $19.3 \pm 3.1$ \\
\hline \multirow[t]{2}{*}{ [S II] 6731} & $23.1 \pm 3.3$ & $15.9 \pm 3.2$ & $6.0 \pm 1.7$ & $5.8 \pm 2.2$ & $11.2 \pm 1.6$ & $10.9 \pm 2.3$ \\
\hline & \multicolumn{2}{|c|}{ IC 1613 H II\#37 } & \multicolumn{2}{|c|}{ IC 2032 ap1 } & \multicolumn{2}{|c|}{ IC 5152 H II\#A } \\
\hline Identification $(\AA)$ & $F$ & $I$ & $F$ & $I$ & $F$ & $I$ \\
\hline [O II] 3727 & $61.0 \pm 1.6$ & $70.1 \pm 7.7$ & $320 \pm 28$ & $286 \pm 59$ & $220.0 \pm 2.5$ & $211 \pm 22$ \\
\hline He II 3834 + H9 & $6.3 \pm 1.1$ & $7.9 \pm 2.1$ & $\ldots$ & $\ldots$ & $\ldots$ & $\ldots$ \\
\hline [Ne III] 3869 & $47.7 \pm 1.4$ & $53.5 \pm 6.0$ & $55 \pm 14$ & $49 \pm 18$ & $21.3 \pm 2.7$ & $20.3 \pm 3.8$ \\
\hline He I 3889 + H8 & $15.6 \pm 1.2$ & $18.0 \pm 2.7$ & $\ldots$ & $\ldots$ & $12.5 \pm 2.1$ & $16.9 \pm 4.7$ \\
\hline$[\mathrm{Ne}$ III] $3967+\mathrm{H} \epsilon$ & $31.6 \pm 1.1$ & $35.2 \pm 4.1$ & $\ldots$ & $\ldots$ & $11.7 \pm 2.0$ & $16.4 \pm 4.7$ \\
\hline $\mathrm{H} \delta 4101$ & $20.16 \pm 0.89$ & $22.3 \pm 2.7$ & $\ldots$ & $\ldots$ & $18.3 \pm 1.8$ & $22.5 \pm 4.2$ \\
\hline $\mathrm{H} \gamma 4340$ & $47.52 \pm 0.84$ & $50.4 \pm 5.4$ & $\ldots$ & $\ldots$ & $42.1 \pm 1.3$ & $45.1 \pm 5.2$ \\
\hline [O III] 4363 & $13.83 \pm 0.70$ & $14.5 \pm 1.8$ & $\ldots$ & $\ldots$ & $3.6 \pm 1.1$ & $3.4 \pm 1.2$ \\
\hline He I 4472 & $\ldots^{a}$ & $\ldots$ & $\ldots$ & $\ldots$ & $5.98 \pm 0.89$ & $5.7 \pm 1.2$ \\
\hline He II 4686 & $21.45 \pm 0.85$ & $21.7 \pm 2.5$ & $\ldots$ & $\ldots$ & $\ldots$ & $\ldots$ \\
\hline He I 4713 & $4.31 \pm 0.68$ & $4.35 \pm 0.96$ & $\ldots$ & $\ldots$ & $\ldots$ & $\ldots$ \\
\hline [Ar IV] 4740 & $2.01 \pm 0.68$ & $2.02 \pm 0.80$ & $\ldots$ & $\ldots$ & $\ldots$ & $\ldots$ \\
\hline $\mathrm{H} \beta 4861$ & $100.0 \pm 1.6$ & $100.0 \pm 5.3$ & $100.0 \pm 9.4$ & $100 \pm 11$ & $100.0 \pm 1.3$ & $100 \pm 5.3$ \\
\hline [O III] 4959 & $176.2 \pm 4.4$ & $174 \pm 19$ & $79.0 \pm 7.6$ & $70 \pm 15$ & $98.1 \pm 3.4$ & $94 \pm 11$ \\
\hline [O III] 5007 & $531.6 \pm 5.5$ & $523 \pm 55$ & $213.9 \pm 9.3$ & $189 \pm 32$ & $294.3 \pm 4.2$ & $282 \pm 30$ \\
\hline He I 5876 & $6.94 \pm 0.68$ & $6.4 \pm 1.1$ & $\ldots$ & $\ldots$ & $12.7 \pm 1.3$ & $12.1 \pm 2.0$ \\
\hline [O I] 6300 & $4.35 \pm 0.77$ & $3.95 \pm 0.94$ & $\ldots$ & $\ldots$ & $5.59 \pm 0.76$ & $5.4 \pm 1.1$ \\
\hline [S III] 6312 & $1.11 \pm 0.67$ & $1.01 \pm 0.67$ & $\ldots$ & $\ldots$ & $\ldots$ & $\ldots$ \\
\hline [O I] 6363 & $1.78 \pm 0.59$ & $1.62 \pm 0.63$ & $\ldots$ & $\ldots$ & $1.38 \pm 0.60$ & $1.32 \pm 0.65$ \\
\hline [N II] 6548 & $4.6 \pm 2.2$ & $4.1 \pm 2.3$ & $\ldots$ & $\ldots$ & $11.4 \pm 2.8$ & $10.9 \pm 3.3$ \\
\hline $\mathrm{H} \alpha 6563$ & $307.0 \pm 2.8$ & $277 \pm 29$ & $316.6 \pm 8.94$ & $286 \pm 46$ & $270.8 \pm 3.5$ & $262 \pm 27$ \\
\hline [N II] 6583 & $4.7 \pm 2.3$ & $4.2 \pm 2.3$ & $19.8 \pm 7.2$ & $17.4 \pm 8.4$ & $20.8 \pm 2.9$ & $20.0 \pm 4.0$ \\
\hline He I 6678 & $2.29 \pm 0.46$ & $2.05 \pm 0.53$ & $\ldots$ & $\ldots$ & $4.54 \pm 0.53$ & $4.34 \pm 0.78$ \\
\hline [S II] 6716 & $12.93 \pm 0.54$ & $11.6 \pm 1.4$ & $48.3 \pm 8.3$ & $42 \pm 12$ & $19.93 \pm 0.59$ & $19.1 \pm 2.1$ \\
\hline [S II] 6731 & $8.45 \pm 0.50$ & $7.55 \pm 0.99$ & $42.9 \pm 8.3$ & $38 \pm 12$ & $14.35 \pm 0.56$ & $13.7 \pm 1.6$ \\
\hline
\end{tabular}

There is currently no velocity listed for this galaxy in the NED database. From the emission lines in the spectrum, the heliocentric velocity was estimated to be $1168 \mathrm{~km} \mathrm{~s}^{-1}$. Assuming that A0355-465 is isolated and that the Hubble constant has a value of $75 \mathrm{~km} \mathrm{~s}^{-1} \mathrm{Mpc}^{-1}$, the estimated distance is 15.6 Mpc and the distance modulus is 31.0. This distance is comparable to that of the Virgo Cluster and the Fornax Cluster. The estimated absolute magnitude in $B$ is -14.7 , which is similar to that of the Local Group dI NGC 6822. The angular dimensions from the NED database are 2.3 by 1.4 , corresponding to linear dimensions $10.4 \mathrm{kpc}$ by $6.3 \mathrm{kpc}$. This is comparable to the dimensions of the Magellanic dwarf NGC 4532 (VCC 1554) in the Virgo Cluster (Hoffman et al. 1999).

An upper limit to the total $21-\mathrm{cm}$ flux was obtained by Longmore et al. (1982), though another measurement should be obtained for confirmation. Nevertheless, A0355-465 exhibits one of the lowest $M_{\mathrm{HI}} / L_{B}$ in the present sample. This dwarf lies
9'.6 away from the face-on SB(rs)cd galaxy NGC 1493, which has a measured heliocentric velocity of $1054 \mathrm{~km} \mathrm{~s}^{-1}$, roughly similar to our estimated velocity for A0355-465. In fact, the dwarf may be a member of a very small group of galaxies with at least four catalogued members (LGG 106; Garcia 1993).

\subsubsection{A1243-335 (ESO381-G020)}

Webster et al. (1983) identified three H II regions in this Cen A group dwarf irregular galaxy. The brightest (labelled \#A) was found in the southeast corner of the galaxy; H II regions \#B and $\mathrm{ZC}$ were found in the northwest and southwest part of the galaxy, respectively. They obtained spectroscopy for all three HII regions and detected [O III] $\lambda 4363$ only in H II \#A. While their quoted uncertainty for the [O III] $\lambda 4363$ detection is rather large $(>40 \%)$, their derived oxygen abundance was 
Table 7. Observed and corrected line ratios for other southern dwarf galaxies; same comments as Table 4.

\begin{tabular}{|c|c|c|c|c|c|c|c|c|}
\hline \multirow[b]{2}{*}{ Identification $(\AA ̊)$} & \multicolumn{2}{|c|}{ NGC 2915 ap1 } & \multicolumn{2}{|c|}{ NGC 2915 ap2 } & \multicolumn{2}{|c|}{ NGC 3109 H II\#6 ap1 } & \multicolumn{2}{|c|}{ NGC 3109 H II\#6 ap2 } \\
\hline & $F$ & $I$ & $F$ & $I$ & $F$ & $I$ & $F$ & $I$ \\
\hline [O II] 3727 & $396 \pm 15$ & $660 \pm 110$ & $306 \pm 35$ & $440 \pm 110$ & $178 \pm 26$ & $152 \pm 60$ & $65 \pm 16$ & $51 \pm 25$ \\
\hline $\mathrm{H} \beta 4861$ & $100.0 \pm 7.5$ & $100 \pm 12$ & $100.0 \pm 9.4$ & $100 \pm 12$ & $100 \pm 18$ & $100 \pm 23$ & $100 \pm 17$ & $100 \pm 23$ \\
\hline [O III] 4959 & $198.9 \pm 7.7$ & $115 \pm 20$ & $63 \pm 10$ & $54 \pm 15$ & $36 \pm 18$ & $30 \pm 23$ & $91 \pm 11$ & $72 \pm 26$ \\
\hline [O III] 5007 & $549.6 \pm 9.2$ & $310 \pm 50$ & $216 \pm 13$ & $181 \pm 34$ & $166 \pm 24$ & $142 \pm 56$ & $200 \pm 13$ & $157 \pm 50$ \\
\hline [N II] 6548 & $22.1 \pm 6.5$ & $6.7 \pm 2.8$ & $11.4 \pm 8.2$ & $7.0 \pm 5.9$ & $\ldots$ & $\ldots$ & $\ldots$ & $\ldots$ \\
\hline $\mathrm{H} \alpha 6563$ & $886.6 \pm 8.1$ & $286 \pm 45$ & $447 \pm 10$ & $286 \pm 47$ & $264 \pm 18$ & $237 \pm 78$ & $300 \pm 17$ & $248 \pm 78$ \\
\hline [N II] 6583 & $74.8 \pm 6.6$ & $22.4 \pm 4.8$ & $64.2 \pm 8.4$ & $39 \pm 10$ & $\ldots$ & $\ldots$ & $\ldots$ & $\ldots$ \\
\hline [S II] 6716 & $101.7 \pm 5.5$ & $29.4 \pm 5.4$ & $73.6 \pm 7.6$ & $44 \pm 10$ & $\ldots$ & $\ldots$ & $\ldots$ & $\ldots$ \\
\hline \multirow[t]{2}{*}{ [S II] 6731} & $70.2 \pm 5.3$ & $20.2 \pm 4.1$ & $32.5 \pm 6.8$ & $19.4 \pm 6.4$ & $\ldots$ & $\ldots$ & $\ldots$ & $\ldots$ \\
\hline & \multicolumn{2}{|c|}{ NGC 3109 H II\#6 ap3 } & \multicolumn{2}{|c|}{ NGC 3109 H II\#6 ap4 } & \multicolumn{2}{|c|}{ NGC 3109 H II\#6 ap5 } & & \\
\hline Identification $(\AA)$ & $F$ & $I$ & $F$ & $I$ & $F$ & $I$ & & \\
\hline [O II] 3727 & $110.5 \pm 8.6$ & $106 \pm 20$ & $108.9 \pm 3.7$ & $109 \pm 16$ & $115.6 \pm 3.8$ & $121 \pm 18$ & & \\
\hline [Ne III] $3967+\mathrm{H} \epsilon$ & $\ldots$ & $\ldots$ & $15.2 \pm 3.8$ & $18.1 \pm 7.0$ & $15.8 \pm 4.1$ & $19.3 \pm 7.6$ & & \\
\hline $\mathrm{H} \delta 4101$ & $\ldots$ & $\ldots$ & $13.1 \pm 2.9$ & $16.8 \pm 6.2$ & $15.8 \pm 2.8$ & $19.7 \pm 5.9$ & & \\
\hline $\mathrm{H} \gamma 4340$ & & & $45.8 \pm 4.0$ & $49.5 \pm 9.7$ & $47.3 \pm 2.9$ & $51.3 \pm 8.8$ & & \\
\hline H $\beta 4861$ & $100.0 \pm 5.2$ & $100.0 \pm 8.9$ & $100.0 \pm 2.9$ & $100.0 \pm 7.5$ & $100.0 \pm 2.6$ & $100.0 \pm 7.3$ & & \\
\hline [O III] 4959 & $59.4 \pm 5.2$ & $54 \pm 11$ & $94.2 \pm 3.1$ & $86 \pm 13$ & $82.4 \pm 2.8$ & $77 \pm 11$ & & \\
\hline [O III] 5007 & $195.4 \pm 6.4$ & $178 \pm 29$ & $289.5 \pm 3.8$ & $265 \pm 38$ & $251.5 \pm 3.4$ & $234 \pm 33$ & & \\
\hline He I 5876 & $16.4 \pm 5.0$ & $14.7 \pm 5.9$ & $14.2 \pm 2.9$ & $12.5 \pm 3.6$ & $13.7 \pm 2.7$ & $12.2 \pm 3.5$ & & \\
\hline [N II] 6548 & & & $3.4 \pm 3.1$ & $2.9 \pm 2.9$ & $7.2 \pm 2.7$ & $6.3 \pm 2.8$ & & \\
\hline $\mathrm{H} \alpha 6563$ & $318.3 \pm 5.6$ & $286 \pm 45$ & $320.9 \pm 3.8$ & $286 \pm 41$ & $323.5 \pm 3.3$ & $286 \pm 40$ & & \\
\hline [N II] 6583 & $15.1 \pm 4.5$ & $13.4 \pm 5.3$ & $14.7 \pm 3.1$ & $12.7 \pm 3.8$ & $16.7 \pm 2.7$ & $14.5 \pm 3.6$ & & \\
\hline [S II] 6716 & $23.4 \pm 2.8$ & $20.6 \pm 4.7$ & $22.9 \pm 1.7$ & $19.8 \pm 3.4$ & $22.5 \pm 1.5$ & $19.5 \pm 3.3$ & & \\
\hline \multirow[t]{2}{*}{ [S II] 6731} & $14.9 \pm 2.6$ & $13.2 \pm 3.7$ & $13.8 \pm 1.5$ & $11.9 \pm 2.4$ & $14.1 \pm 1.4$ & $12.1 \pm 2.4$ & & \\
\hline & \multicolumn{2}{|c|}{ NGC 3109 H II\#6 ap6 } & \multicolumn{2}{|c|}{ NGC 3109 H II\#6 ap7 } & \multicolumn{2}{|c|}{ Sag DIG H II\#3 } & & \\
\hline Identification $(\AA)$ & $F$ & $I$ & $F$ & $I$ & $F$ & $I$ & & \\
\hline [O II] 3727 & $118.8 \pm 4.0$ & $129 \pm 19$ & $204 \pm 27$ & $270 \pm 100$ & $71.5 \pm 6.9$ & $95 \pm 17$ & & \\
\hline [Ne III] $3967+\mathrm{H} \epsilon$ & $14.3 \pm 3.8$ & $18.0 \pm 6.3$ & $\ldots$ & $\ldots$ & $\ldots$ & $\ldots$ & & \\
\hline $\mathrm{H} \delta 4101$ & $18.9 \pm 3.2$ & $22.7 \pm 6.4$ & $\ldots$ & $\ldots$ & $17.5 \pm 3.3$ & $24.6 \pm 7.3$ & & \\
\hline $\mathrm{H} \gamma 4340$ & $46.4 \pm 3.9$ & $50.4 \pm 9.6$ & $\ldots$ & $\ldots$ & $48.2 \pm 4.4$ & $57 \pm 10$ & & \\
\hline $\mathrm{H} \beta 4861$ & $100.0 \pm 3.2$ & $100.0 \pm 7.6$ & $100 \pm 18$ & $100 \pm 22$ & $100.0 \pm 3.8$ & $100.0 \pm 6.5$ & & \\
\hline [O III] 4959 & $67.4 \pm 3.0$ & $65 \pm 10$ & $31 \pm 16$ & $28 \pm 21$ & $54.1 \pm 4.0$ & $51.5 \pm 8.0$ & & \\
\hline [O III] 5007 & $203.2 \pm 3.6$ & $195 \pm 28$ & $111 \pm 19$ & $98 \pm 40$ & $165.8 \pm 5.0$ & $157 \pm 19$ & & \\
\hline He I 5876 & $12.7 \pm 2.3$ & $11.7 \pm 3.2$ & $\ldots$ & $\ldots$ & $\ldots$ & $\ldots$ & & \\
\hline [N II] 6548 & $11.3 \pm 2.5$ & $10.1 \pm 3.1$ & $\ldots$ & $\ldots$ & $4.4 \pm 3.2$ & $3.4 \pm 2.7$ & & \\
\hline $\mathrm{H} \alpha 6563$ & $318.3 \pm 3.2$ & $286 \pm 41$ & $407 \pm 17$ & $286 \pm 86$ & $369.1 \pm 4.1$ & $286 \pm 33$ & & \\
\hline [N II] 6583 & $18.4 \pm 2.6$ & $16.4 \pm 3.8$ & $46 \pm 14$ & $32 \pm 17$ & $3.5 \pm 3.3$ & $2.7 \pm 2.7$ & & \\
\hline [S II] 6716 & $23.5 \pm 1.6$ & $20.9 \pm 3.6$ & $\ldots$ & $\ldots$ & $18.5 \pm 5.6$ & $14.1 \pm 5.3$ & & \\
\hline [S II] 6731 & $14.9 \pm 1.5$ & $13.3 \pm 2.6$ & $\ldots$ & $\ldots$ & $9.7 \pm 4.4$ & $7.4 \pm 3.9$ & & \\
\hline
\end{tabular}

$12+\log (\mathrm{O} / \mathrm{H})=8.04$. H II regions \#A and \#B appear to be spatially coincident with local maxima in H I (Côté et al. 2000). The H I extent is 4.5 times larger than the optical galaxy.

Our spectrum of H II region \#A revealed the bright oxygen lines, but no [O III] $\lambda 4363$. The derived bright-line oxygen abundance is $12+\log (\mathrm{O} / \mathrm{H})=7.78$, which is almost 0.3 dex lower than the Webster et al. (1983) value. The adopted nitrogen-to-oxygen abundance ratio is $\log (\mathrm{N} / \mathrm{O})=-1.62$.

\subsubsection{A1324-412 (ESO324-G024)}

For this Cen A group dwarf irregular, Côté et al. (1997) measured the total HI flux, and Karachentsev et al. (2002b) derived a TRGB distance. However, our spectrum only revealed $\mathrm{H} \beta$ and $\mathrm{H} \alpha$ emission; the subsequent derived reddening is $E(B-V)=+0.14$ mag.

\subsubsection{A1334-277 (ESO444-G084)}

The oxygen abundance reported here $(12+\log (\mathrm{O} / \mathrm{H})=7.40)$ is the first ever published for this Cen A group member and is the third most metal-poor galaxy in the present study. While the upper limit to the $[\mathrm{N} \mathrm{II}] /[\mathrm{O} \mathrm{II}]$ intensity ratio may indicate an upper branch abundance for this Cen A group dwarf, a low metallicity is assumed, because the $I([\mathrm{~N} \mathrm{II}] \lambda 6583) / I(\mathrm{H} \alpha)$ and $I([\mathrm{~S} \mathrm{II}] \lambda 6716) / I(\mathrm{H} \alpha)$ ratios are small, i.e., less than $3 \%$ and $2 \%$, respectively. Indeed, bright-line calibrations using $I([\mathrm{~N} \mathrm{II}] \lambda 6583) / I(\mathrm{H} \alpha)$ (van Zee et al. 1998b; Denicoló et al. 2002) yield lower branch abundances.

While this dwarf galaxy is relatively metal-poor, it is not particularly isolated. The TRGB distance of this $\mathrm{dI}$ is $4.61 \mathrm{Mpc}$, which puts this galaxy $380 \mathrm{kpc}$ from the spiral galaxy M 83 (Karachentsev et al. 2002b). While the H I extent is almost six times larger than the optical extent (Côté et al. 2000), the $M_{\mathrm{HI}} / L_{B}$ ratio is normal for its luminosity compared to typical dIs (e.g., Roberts \& Haynes 1994; Skillman 1996; Pildis et al. 1997).

\subsubsection{A1346-358 (ESO383-G087)}

Webster et al. (1983) obtained spectroscopy of three H II regions (A, B, and C), but did not detect [O III] $\lambda 4363$ at all. They estimated the oxygen abundance to be $12+\log (\mathrm{O} / \mathrm{H}) \approx 8.3$. In our spectrum of $\mathrm{HII}$ region \#A, [O III] $\lambda 4363$ was 
Table 8. Derived properties. Column (1): H II region. Column (2): H $\beta$ intensity, corrected for underlying Balmer absorption and adopted reddening. Columns (3) and (4): Computed and adopted reddening values. Column (5): Observed $\mathrm{H} \beta$ emission equivalent width. Column (6): Electron density. Column (7): $\mathrm{O}^{+2}$ electron temperature arising from [O III] $] 4363$ detection or upper limit. Column (8): $\mathrm{O}^{+}$electron temperature. NOTE: ${ }^{a}$ Background galaxy with $v_{\odot} / c \simeq+0.11$. Observed (uncorrected) $\mathrm{H} \beta$ flux and $\mathrm{H} \beta$ emission equivalent width are listed.

\begin{tabular}{|c|c|c|c|c|c|c|c|}
\hline $\begin{array}{l}\text { H II Region } \\
\text { (1) }\end{array}$ & $\begin{array}{c}I(\mathrm{H} \beta) \\
\left(\operatorname{ergs~s}^{-1} \mathrm{~cm}^{-2}\right) \\
(2)\end{array}$ & $\begin{array}{c}\text { Derived } \\
E(B-V) \\
(\mathrm{mag}) \\
(3)\end{array}$ & $\begin{array}{c}\text { Adopted } \\
E(B-V) \\
(\mathrm{mag}) \\
(4)\end{array}$ & $\begin{array}{c}W_{\mathrm{em}}(\mathrm{H} \beta) \\
(\AA) \\
(5)\end{array}$ & $\begin{array}{c}n_{e} \\
\left(\mathrm{~cm}^{-3}\right) \\
(6)\end{array}$ & $\begin{array}{c}T_{e}\left(\mathrm{O}^{+2}\right) \\
(\mathrm{K}) \\
(7)\end{array}$ & $\begin{array}{c}T_{e}\left(\mathrm{O}^{+}\right) \\
(\mathrm{K}) \\
(8)\end{array}$ \\
\hline \multicolumn{8}{|c|}{ Centaurus A group dwarfs } \\
\hline A1243-335 H II\#A & $(4.44 \pm 0.54) \times 10^{-16}$ & $+0.14 \pm 0.16$ & +0.14 & $18.3 \pm 2.1$ & 100 & $\ldots$ & $\ldots$ \\
\hline A1324-412 ap1 & $(1.88 \pm 0.34) \times 10^{-15}$ & $+0.14 \pm 0.14$ & +0.14 & $14.7 \pm 2.4$ & 100 & $\ldots$ & $\ldots$ \\
\hline A1334-277 ap1 & $(1.15 \pm 0.19) \times 10^{-16}$ & $-0.07 \pm 0.25$ & 0 & $17.2 \pm 2.5$ & 100 & $\ldots$ & $\ldots$ \\
\hline A1346-358 H II\#A & $(7.73 \pm 0.34) \times 10^{-15}$ & $+0.427 \pm 0.087$ & +0.427 & $103.7 \pm 4.3$ & 100 & 10935 & 11504 \\
\hline DDO 161 ap1 & $(3.71 \pm 0.18) \times 10^{-15}$ & $+0.399 \pm 0.091$ & +0.399 & $43.6 \pm 1.4$ & 31 & $<15460$ & $<13970$ \\
\hline NGC 5264 ap1 & $(5.66 \pm 0.52) \times 10^{-16}$ & $+0.15 \pm 0.14$ & +0.15 & $11.09 \pm 0.84$ & 100 & $\ldots$ & $\ldots$ \\
\hline \multicolumn{8}{|c|}{ Sculptor group dwarfs } \\
\hline AM0106-382 ap1 & $(3.30 \pm 0.40) \times 10^{-16}$ & $\ldots$ & 0 & $24.3 \pm 2.7$ & 100 & $\ldots$ & $\ldots$ \\
\hline AM0106-382 ap2 & $(9.28 \pm 0.82) \times 10^{-16}$ & $\ldots$ & 0 & $18.4 \pm 1.3$ & 100 & $\ldots$ & $\ldots$ \\
\hline AM0106-382 ap3 & $(5.86 \pm 0.52) \times 10^{-16}$ & $\ldots$ & 0 & $15.4 \pm 1.0$ & 100 & $\ldots$ & $\ldots$ \\
\hline ESO347-G017 ap1 & $(2.46 \pm 0.14) \times 10^{-15}$ & $+0.02 \pm 0.11$ & +0.02 & $17.91 \pm 0.45$ & 100 & $<13680$ & $<13090$ \\
\hline ESO347-G017 ap2 & $(2.33 \pm 0.15) \times 10^{-15}$ & $+0.14 \pm 0.12$ & +0.14 & $14.86 \pm 0.54$ & 100 & $\ldots$ & $\ldots$ \\
\hline ESO347-G017 ap3 & $(8.1 \pm 1.0) \times 10^{-16}$ & $+0.25 \pm 0.19$ & +0.25 & $12.5 \pm 1.3$ & 100 & $\ldots$ & $\ldots$ \\
\hline ESO348-G009 ap1 & $(9.8 \pm 1.4) \times 10^{-16}$ & $\ldots$ & 0 & $16.6 \pm 2.2$ & 100 & $\ldots$ & $\ldots$ \\
\hline ESO348-G009 ap $2^{a}$ & $(2.32 \pm 0.76) \times 10^{-15}$ & $\ldots$ & $\ldots$ & $5.2 \pm 1.7$ & $\ldots$ & $\ldots$ & $\ldots$ \\
\hline UGCA 442 H II\#2 & $(2.23 \pm 0.13) \times 10^{-15}$ & $+0.13 \pm 0.11$ & +0.13 & $17.08 \pm 0.47$ & 100 & $<17480$ & $<14830$ \\
\hline \multicolumn{8}{|c|}{ Other southern dwarfs } \\
\hline A0355-465 H II\#B & $(2.70 \pm 0.14) \times 10^{-15}$ & $+0.318 \pm 0.095$ & +0.318 & $50.2 \pm 2.3$ & 100 & $\ldots$ & $\ldots$ \\
\hline ESO358-G060 ap1 & $(1.35 \pm 0.11) \times 10^{-15}$ & $-0.13 \pm 0.15$ & 0 & $56.8 \pm 4.3$ & 100 & $<18160$ & $<15090$ \\
\hline IC 1613 H II\#13 & $(4.25 \pm 0.18) \times 10^{-15}$ & $\ldots$ & 0 & $89.1 \pm 4.6$ & 100 & $<14540$ & $<16760$ \\
\hline IC 1613 H II\#37 & $(1.275 \pm 0.068) \times 10^{-14}$ & $+0.10 \pm 0.11$ & +0.10 & $514 \pm 97$ & 100 & 17910 & 14990 \\
\hline IC 2032 ap1 & $(1.94 \pm 0.22) \times 10^{-16}$ & $+0.01 \pm 0.16$ & +0.01 & $15.0 \pm 1.5$ & 100 & $\ldots$ & $\ldots$ \\
\hline IC 5152 H II\#A & $(1.732 \pm 0.091) \times 10^{-14}$ & $\ldots$ & 0 & $44.90 \pm 0.85$ & 37 & 12360 & 12360 \\
\hline NGC 2915 ap1 & $(1.84 \pm 0.21) \times 10^{-14}$ & $+0.72 \pm 0.16$ & +0.72 & $4.94 \pm 0.26$ & 100 & $\ldots$ & $\ldots$ \\
\hline NGC 2915 ap2 & $(7.62 \pm 0.88) \times 10^{-16}$ & $+0.36 \pm 0.17$ & +0.36 & $13.8 \pm 1.4$ & 100 & $\ldots$ & $\ldots$ \\
\hline NGC 3109 H II\#6 ap1 & $(2.67 \pm 0.59) \times 10^{-16}$ & $-0.19 \pm 0.33$ & 0 & $11.9 \pm 2.3$ & 100 & $\ldots$ & $\ldots$ \\
\hline NGC 3109 H II\#6 ap2 & $(2.99 \pm 0.65) \times 10^{-16}$ & $-0.15 \pm 0.31$ & 0 & $7.3 \pm 1.2$ & 100 & $\ldots$ & $\ldots$ \\
\hline NGC 3109 H II\#6 ap3 & $(9.91 \pm 0.83) \times 10^{-16}$ & $+0.03 \pm 0.16$ & +0.03 & $21.0 \pm 1.2$ & 100 & $\ldots$ & $\ldots$ \\
\hline NGC 3109 H II\#6 ap4 & $(2.46 \pm 0.18) \times 10^{-15}$ & $+0.06 \pm 0.14$ & +0.06 & $23.25 \pm 0.80$ & 100 & $\ldots$ & $\ldots$ \\
\hline NGC 3109 H II\#6 ap5 & $(4.58 \pm 0.33) \times 10^{-15}$ & $+0.08 \pm 0.14$ & +0.08 & $31.3 \pm 1.1$ & 100 & $\ldots$ & $\ldots$ \\
\hline NGC 3109 H II\#6 ap6 & $(1.92 \pm 0.14) \times 10^{-15}$ & $+0.08 \pm 0.14$ & +0.08 & $67.5 \pm 4.5$ & 100 & $\ldots$ & $\ldots$ \\
\hline NGC 3109 H II\#6 ap7 & $(6.2 \pm 1.3) \times 10^{-16}$ & $+0.28 \pm 0.30$ & +0.28 & $20.7 \pm 4.3$ & 100 & $\ldots$ & $\ldots$ \\
\hline Sag DIG H II\#3 & $(8.82 \pm 0.57) \times 10^{-16}$ & $+0.23 \pm 0.12$ & +0.23 & $67.0 \pm 4.8$ & 100 & $\ldots$ & $\ldots$ \\
\hline
\end{tabular}

detected and the subsequent oxygen abundance is $12+\log (\mathrm{O} / \mathrm{H})$ $=8.19 \pm 0.06$, or about one-fifth of the solar value. The nitrogenand neon-to-oxygen ratios are: $\log (\mathrm{N} / \mathrm{O})=-1.37 \pm 0.08$ and $\log (\mathrm{Ne} / \mathrm{O})=-0.46 \pm 0.05$. The latter is about 0.2 dex larger than values obtained for blue compact dwarf galaxies at similar oxygen abundance (Izotov \& Thuan 1999). This dwarf lies (in projection) approximately between the elliptical Cen A and the spiral M 83. The proximity to these two giant galaxies may explain why the $M_{\mathrm{HI}} / L_{B}$ value for the dwarf is several times lower than typical values for dIs at comparable luminosities (e.g., Staveley-Smith et al. 1992).

\subsubsection{AM0106-382}

Aside from the properties listed in Table 1, and the inclusion of this galaxy in the H I catalog by Côté et al. (1997), there is little in the literature describing further this dwarf irregular in the Sculptor group. The adopted oxygen abundance is $12+\log (\mathrm{O} / \mathrm{H})=7.60$ and the nitrogen-to-oxygen ratio is $\log (\mathrm{N} / \mathrm{O})=-1.42$. These values are the first ever published for this galaxy.

\subsubsection{DDO 161}

This Cen A group dwarf galaxy was studied in some detail by Karachentsev et al. (1981), but the oxygen abundance here is believed to be the first ever published for this galaxy. The $\mathrm{HI}$ extent is almost five times larger than the optical extent (Côté et al. 2000). The spectrum presented here was taken with the east-west slit placed so that the most northern starforming clump was observed. The adopted oxygen abundance is $12+\log (\mathrm{O} / \mathrm{H})=8.06$ and the nitrogen-to-oxygen ratio is $\log (\mathrm{N} / \mathrm{O})=-1.91$.

\subsubsection{ESO302-G014 (AM0349-383)}

Little is described of this galaxy in the literature. Because only [O III] $\lambda 5007$ and $\mathrm{H} \alpha$ were detected in the spectrum, there is no subsequent analysis. 
Table 9. Derived nebular abundances. Column (1): H II region. Column (2): Oxygen abundance from [O III] $\lambda 4363$ measurements; lower limits to the oxygen abundance were obtained from $2 \sigma$ upper limits to the [O III] $\lambda 4363$ flux. Column (3): Oxygen abundance d. Column (4): Oxygen abundance derived using bright-line method by Pilyugin (2000). Columns (5) and (6): Nitrogen-to-oxygen abundance ratios: derived with $\mathrm{O}^{+}$ temperatures from Table 8 and with the bright-line method, respectively. Columns (7) and (8): Neon-to-oxygen abundance ratios: derived with $\mathrm{O}^{+2}$ temperatures from Table 8 and with the bright-line method, respectively. NOTES: ${ }^{a}$ The Pilyugin value is uncertain, but upper branch abundances appear to be correct; see text in Sect. 6.1.16. ${ }^{b}$ Computed using the method of Thurston et al. (1996). ${ }^{c} I([\mathrm{~N}$ II $] \lambda \lambda 6548,6583)$ is likely a lower limit. ${ }^{d} I([\mathrm{Ne} \mathrm{III}] \lambda 3869)$ likely too low. ${ }^{e}$ Pilyugin value is uncertain. Oxygen abundances derived with $I([\mathrm{~N}$ II $] \lambda 6583) / I(\mathrm{H} \alpha)$ calibrations (van Zee et al. 1998b; Denicoló et al. 2002) are consistent with lower branch values; the value listed here is the average. ${ }^{f}[\mathrm{~N}$ II] $\lambda 6583$ not measured; cannot break degeneracy in bright-line method. ${ }^{g}$ Same as note $e$, but see also Sect. 6.1.15.

\begin{tabular}{|c|c|c|c|c|c|c|c|}
\hline \multirow[b]{3}{*}{$\begin{array}{l}\text { H II Region } \\
\text { (1) }\end{array}$} & \multicolumn{3}{|c|}{$12+\log (\mathrm{O} / \mathrm{H})$} & \multirow{2}{*}{\multicolumn{2}{|c|}{$\log (\mathrm{N} / \mathrm{O})$}} & \multirow{2}{*}{\multicolumn{2}{|c|}{$\log (\mathrm{Ne} / \mathrm{O})$}} \\
\hline & \multirow{2}{*}{$\begin{array}{c}\text { Direct } \\
{[\mathrm{O} \text { III }] \lambda 4363} \\
(2) \\
\end{array}$} & \multicolumn{2}{|c|}{ Bright-Line } & & & & \\
\hline & & $\begin{array}{l}\text { McGaugh } \\
\text { (3) }\end{array}$ & $\begin{array}{l}\text { Pilyugin } \\
\text { (4) }\end{array}$ & $\begin{array}{l}\text { Direct } \\
(5)\end{array}$ & $\begin{array}{l}\text { Bright-line } \\
\text { (6) }\end{array}$ & $\begin{array}{l}\text { Direct } \\
(7)\end{array}$ & $\begin{array}{c}\text { Bright-line } \\
\text { (8) }\end{array}$ \\
\hline \multicolumn{8}{|c|}{ Centaurus A group dwarfs } \\
\hline A1243-335 H II\#A & $\ldots$ & 7.87 & 7.69 & $\ldots$ & -1.62 & $\ldots$ & $\ldots$ \\
\hline A1334-277 ap1 & $\ldots$ & 7.45 & 7.34 & $\ldots$ & -1.04 & $\ldots$ & $\ldots$ \\
\hline A1346-358 H II\#A & $8.19 \pm 0.06$ & 8.26 & 8.22 & $-1.37 \pm 0.08$ & -1.44 & $-0.46 \pm 0.05$ & -0.50 \\
\hline DDO 161 ap1 & $>7.76$ & 8.08 & 8.03 & $\ldots$ & -1.91 & $\ldots$ & $\ldots$ \\
\hline NGC 5264 ap1 & $\ldots$ & 8.66 & $8.54^{a}$ & $\ldots$ & $-0.57^{b}$ & $\ldots$ & $\ldots$ \\
\hline \multicolumn{8}{|c|}{ Sculptor group dwarfs } \\
\hline AM0106-382 ap1 & $\ldots$ & 7.54 & 7.71 & $\ldots$ & -1.22 & $\ldots$ & $\ldots$ \\
\hline AM0106-382 ap2 & $\ldots$ & 7.58 & 7.56 & $\ldots$ & -1.47 & $\ldots$ & $\ldots$ \\
\hline AM0106-382 ap3 & $\ldots$ & 7.61 & 7.59 & $\ldots$ & -1.58 & $\ldots$ & $\ldots$ \\
\hline ESO347-G017 ap1 & $>7.80$ & 7.89 & 7.78 & $\ldots$ & -1.30 & $\ldots$ & -0.52 \\
\hline ESO347-G017 ap2 & $\ldots$ & 7.92 & 7.76 & $\ldots$ & -1.31 & $\ldots$ & -0.53 \\
\hline ESO347-G017 ap3 & $\ldots$ & 7.96 & 8.03 & $\ldots$ & $>-1.86^{c}$ & $\ldots$ & $\ldots$ \\
\hline ESO348-G009 ap1 & $\ldots$ & 7.89 & 8.07 & $\ldots$ & -1.60 & $\ldots$ & $\ldots$ \\
\hline UGCA 442 H II\#2 & $>7.48$ & 7.81 & 7.88 & $\ldots$ & -1.41 & $\ldots$ & $\ldots$ \\
\hline \multicolumn{8}{|c|}{ Other southern dwarfs } \\
\hline A0355-465 H II\#B & $\ldots$ & 8.23 & 8.01 & $\ldots$ & -1.61 & 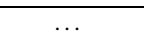 & -0.48 \\
\hline ESO358-G060 ap1 & $>7.26$ & 7.38 & 7.26 & $\ldots$ & -1.24 & $\ldots$ & $>-1.11^{d}$ \\
\hline IC 1613 H II\#13 & $>7.61$ & 7.90 & 7.89 & $\ldots$ & -1.40 & $\ldots$ & $\ldots$ \\
\hline IC 1613 Н II\#37 & $7.62 \pm 0.05$ & 7.88 & 7.71 & $-1.13 \pm 0.18$ & -1.35 & $-0.60 \pm 0.05$ & -0.62 \\
\hline IC 2032 ap1 & $\ldots$ & 7.96 & 7.98 & $\ldots$ & -1.37 & $\ldots$ & -0.21 \\
\hline IC 5152 H II\#A & $7.92 \pm 0.07$ & 7.91 & 7.80 & $-1.05 \pm 0.12$ & -1.09 & $-0.69 \pm 0.08$ & -0.77 \\
\hline NGC 2915 ap1 & $\ldots$ & 8.29 & $8.33^{e}$ & $\ldots$ & -1.71 & $\ldots$ & $\ldots$ \\
\hline NGC 2915 ap2 & $\ldots$ & 8.21 & 8.35 & $\ldots$ & -1.25 & $\ldots$ & $\ldots$ \\
\hline NGC 3109 H II\#6 ap $1^{f}$ & $\ldots$ & $\ldots$ & $\ldots$ & $\ldots$ & $\ldots$ & $\ldots$ & $\ldots$ \\
\hline NGC 3109 H II\#6 ap2 ${ }^{f}$ & $\ldots$ & $\ldots$ & $\ldots$ & $\ldots$ & $\ldots$ & $\ldots$ & $\ldots$ \\
\hline NGC 3109 H II\#6 ap3 ${ }^{g}$ & $\ldots$ & 7.50 & 7.40 & $\ldots$ & -1.37 & $\ldots$ & $\ldots$ \\
\hline NGC 3109 H II\#6 ap4 ${ }^{g}$ & $\ldots$ & 8.07 & 8.13 & $\ldots$ & -1.36 & $\ldots$ & $\ldots$ \\
\hline NGC 3109 H II\#6 ap5 ${ }^{g}$ & $\ldots$ & 7.64 & 7.52 & $\ldots$ & -1.28 & $\ldots$ & $\ldots$ \\
\hline NGC 3109 H II\#6 ap6 ${ }^{g}$ & $\ldots$ & 7.60 & 7.51 & $\ldots$ & -1.20 & $\ldots$ & $\ldots$ \\
\hline NGC 3109 H II\#6 ap7 ${ }^{g}$ & $\ldots$ & 7.85 & 8.08 & $\ldots$ & -1.38 & $\ldots$ & $\ldots$ \\
\hline Sag DIG H II\#3 & $\ldots$ & 7.44 & 7.33 & $\ldots$ & -1.63 & $\ldots$ & $\ldots$ \\
\hline
\end{tabular}

\subsubsection{ESO347-G017, ESO348-G009}

Aside from the properties listed in Table 1, and the inclusion of this galaxy in the H I catalog by Côté et al. (1997), there is little in the literature describing further these two dwarf galaxies in the Sculptor group. The adopted (bright-line) oxygen abundances, respectively, are: $12+\log (\mathrm{O} / \mathrm{H})=7.84$, and 7.98. The nitrogen-to-oxygen ratios, respectively, $\operatorname{are:} \log (\mathrm{N} / \mathrm{O})=-1.49$, and -1.60 . The neon-to-oxygen ratio for ESO347-G017 is $\log (\mathrm{Ne} / \mathrm{O})=-0.53$.

\subsubsection{ESO358-G060 (FCC 302)}

This dwarf is a confirmed member of the Fornax Cluster (FCC 302; Drinkwater et al. 2001; Schröder et al. 2001). With an assumed distance modulus of 31.5 (Mould et al. 2000), the absolute magnitude is $M_{B} \simeq-15.7$. The derived H I mass to blue luminosity ratio is $\simeq 4$, which is the largest in the present sample.
The derived oxygen abundance $(12+\log (\mathrm{O} / \mathrm{H})=7.32$; $\sim 2.8 \%$ of solar) makes this galaxy the most metal-poor dwarf in the present study. The abundance is comparable to the second most metal-poor galaxy known, i.e., the blue compact dwarf galaxy SBS 0335-052 with $12+\log (\mathrm{O} / \mathrm{H})=7.33$ (Melnick et al. 1992; Izotov et al. 1997a). If the abundance is truly low, additional spectroscopy of this dI with a larger telescope should reveal [O III] $\lambda 4363$, which should still be observable even at the distance of $\sim 20 \mathrm{Mpc}$; see Lee et al. (2003b) for [O III] $\lambda 4363$ detections in dwarf galaxies at the distance of the Virgo Cluster. The adopted nitrogen-to-oxygen ratio is $\log (\mathrm{N} / \mathrm{O})=-1.24$.

\subsubsection{IC 1613}

Located in the Local Group, IC 1613 is a well-studied dI whose position is near the celestial equator. The $M_{\mathrm{HI}} / L_{B}$ value for IC 1613 is typical for its luminosity compared to other dIs. Its H II regions have been catalogued by Sandage (1971), 


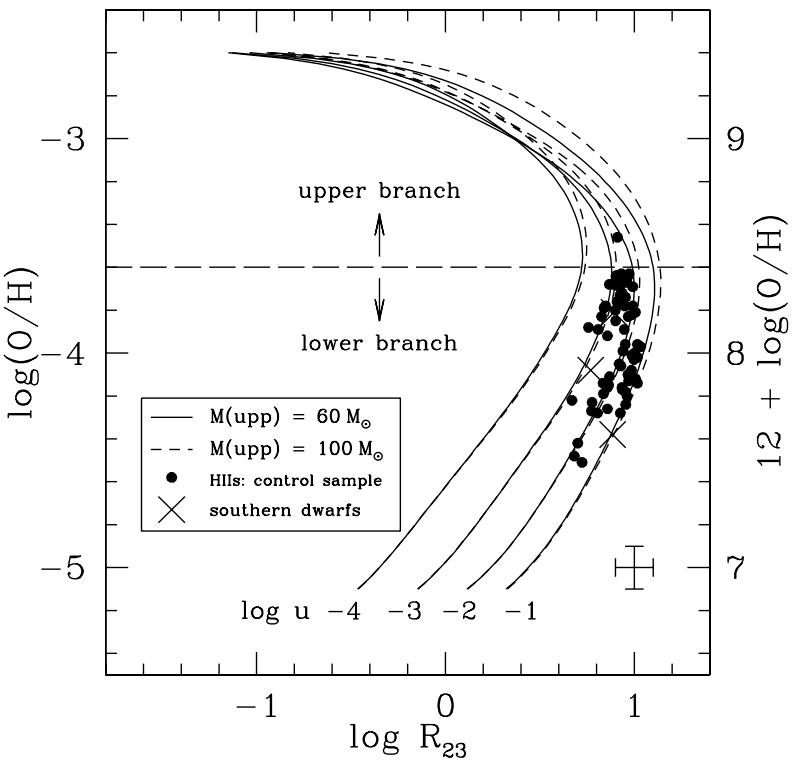

Fig. 3. Oxygen abundance versus bright-line indicator, $R_{23}$. The filled circles indicate H II regions from the control sample of nearby dIs (Lee et al. 2003a), whose oxygen abundances were obtained directly from measurements of the [O III] $\lambda 4363$ emission line. Crosses indicate southern dwarfs in the present sample for which [O III] $\lambda 4363$ was measured. Calibration curves for $\log (\mathrm{O} / \mathrm{H})$ against $\log R_{23}$ (McGaugh 1997, private communication) are plotted for four different values of the ionization parameter $(\log u$; see McGaugh 1991). These curves were derived using a standard stellar initial mass function for a cluster of ionizing stars with an upper mass limit of $\mathcal{M}_{\text {upp }}=60 M_{\odot}$ (solid lines) and $100 M_{\odot}$ (short-dash lines); see also McGaugh (1991). The horizontal long-dash line marks the approximate boundary below (above) which the lower (upper) branch occurs. [O III] $\lambda 4363$ abundances are consistent with lower branch values. The error bars at the lower right indicates typical uncertainties of $0.1 \mathrm{dex}$ for the $R_{23}$ indicator and 0.1 dex for the direct oxygen abundance.

Lequeux et al. (1987), Price et al. (1990), and Hodge et al. (1990). The brightest H II region was identified by Sandage as \#3 (S3), which has been labelled H II \#37 by Hodge et al. (1990). Talent (1980), D'Odorico \& Rosa (1982), and Davidson \& Kinman (1982) obtained photoelectric spectrophotometry of S3, and all three studies showed that the ionization source was a single Wolf-Rayet (W-R) star. Cole et al. (1999) and Dolphin et al. (2001b) obtained resolved stellar photometry and derived distances from the tip of the red giant branch and the red clump, respectively; these distance measurements are in agreement $(730 \mathrm{kpc})$. Rosado et al. (2001) and Valdez-Gutiérrez et al. (2001) obtained Fabry-Perot interferometry at $\mathrm{H} \alpha$ and [S II] $\lambda \lambda 6716,6731$ to study the kinematics of S3 and S8 and found that the nebular emission exhibits twolobe structure with superbubbles covering the entire galaxy.

Talent (1980) and Davidson \& Kinman (1982) obtained [O III] $\lambda 4363$ measurements of HII \#37, and derived $12+\log (\mathrm{O} / \mathrm{H})=7.86$ and 7.87 , respectively, and $\log (\mathrm{N} / \mathrm{O})=-1.21$ (upper limit) and -0.99 , respectively. Hunter \& Gallagher (1985) and Peimbert et al. (1988) measured additional spectrophotometry for other H II regions, of which one was identified as a supernova remnant (H II \#49, Sandage \#8 or S8; D’Odorico et al. 1980;

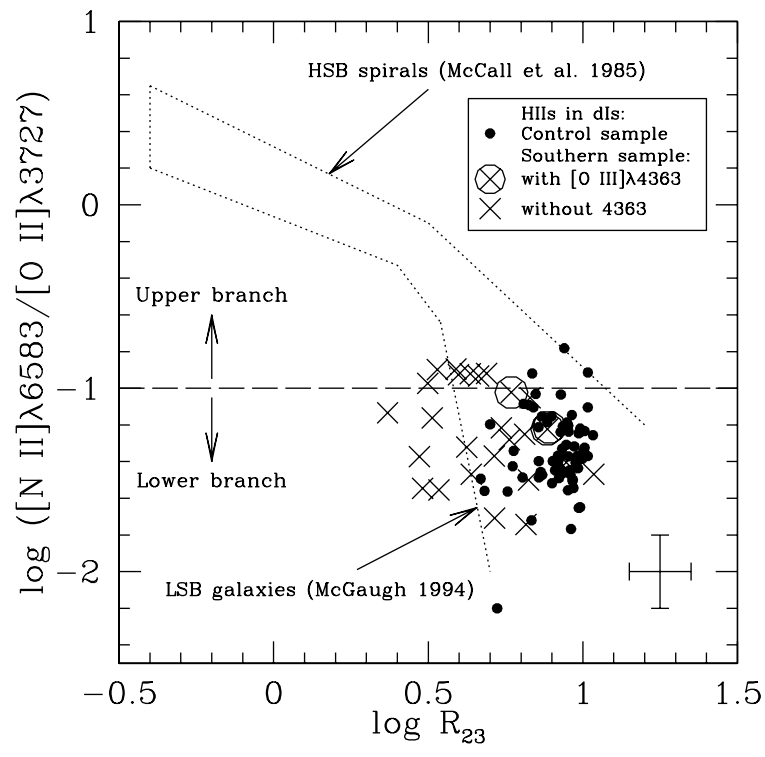

Fig. 4. $[\mathrm{N} \mathrm{II}] /[\mathrm{O} \mathrm{II}]$ discriminant versus bright-line indicator, $R_{23}$. Filled circles indicate H II regions from the control sample of nearby dIs with [O III] $\lambda 4363$ detections (Lee et al. 2003a). Crosses indicate H II regions for the present sample of southern dwarfs; crosses surrounded by open circles indicate [O III] $\lambda 4363$ measurements. The error bars at the lower right indicate typical uncertainties of $0.1 \mathrm{dex}$ for the $R_{23}$ indicator and $0.2 \mathrm{dex}$ (at most) for the [N II]/[O II] ratio. The dotted lines mark the regions in the diagram occupied by high surface brightness (HSB) spiral galaxies (McCall et al. 1985) at the upper left and low surface brightness (LSB) galaxies (McGaugh 1994) towards the lower right. As suggested by McGaugh, the horizontal dashed line marks the approximate boundary below (above) which the lower (upper) branch of the bright-line method is selected to determine a unique value of an oxygen abundance.

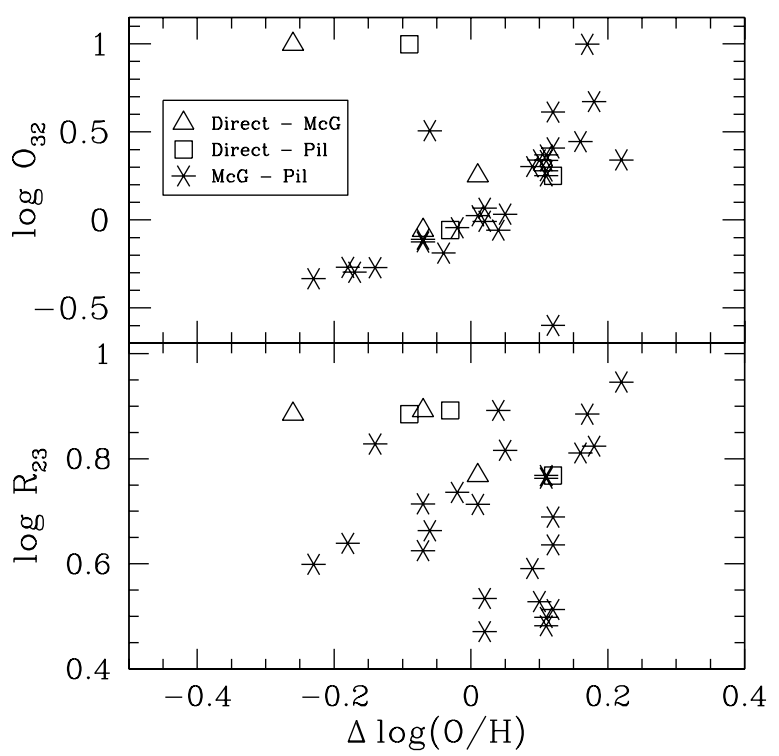

Fig. 5. Difference in oxygen abundance from various methods versus $\log O_{32}$ (top panel), and versus $\log R_{23}$ (bottom panel). Each point represents an H II region from dwarf galaxies in the southern sample. "Direct" denotes oxygen abundances derived from [O III] $\lambda 4363$ measurements, "McG" denotes oxygen abundances derived using the bright-line method by McGaugh (1991), and "Pil" denotes oxygen abundances derived using the bright-line method by Pilyugin (2000, 2001a). 
Rosado et al. 2001). For H II \#49, D'Odorico \& Dopita (1983) derived $12+\log (\mathrm{O} / \mathrm{H})=7.60$ and $\log (\mathrm{N} / \mathrm{O})=-0.90$, while Peimbert et al. (1988) derived $12+\log (\mathrm{O} / \mathrm{H})=7.83$ and $\log (\mathrm{N} / \mathrm{O})=-1.15$.

Our spectrum of HII \#37 (Fig. 2) also exhibits broad Wolf-Rayet features near 4471, 4686, and $5800 \AA$. Due to a strong [O III] $\lambda 4363$ detection, an oxygen abundance of $12+\log (\mathrm{O} / \mathrm{H})=7.62 \pm 0.05$ was derived, which is the adopted value here, and $1.6 \sigma$ lower than the [O III] $\lambda 4363$ abundance (7.70) obtained most recently by Kingsburgh \& Barlow (1995). Our $\log (\mathrm{N} / \mathrm{O})=-1.13 \pm 0.18$ value is in agreement with the value $(-0.96)$ obtained by Kingsburgh \& Barlow (1995). Our adopted neon-to-oxygen ratio is $\log (\mathrm{Ne} / \mathrm{O})=-0.60 \pm 0.05$, which is in rough agreement with determinations by Izotov \& Thuan (1999) for other dwarf galaxies at similar oxygen abundance. A new spectrum for H II \#13 did not reveal [O III] $] 4363$. Oxygen abundances and N/O values derived using the bright-line method for H II regions \#13 and \#37 are in agreement.

\subsubsection{IC 2032}

Not much is known about this dwarf, although it may be a member of the Dorado group; see Carrasco et al. (2001) and references therein. The HI properties of the galaxy have been studied and listed in the survey by Huchtmeier et al. (2000a). There is a bright "shell" of star formation, giving the galaxy a "cometary" appearance. The spectrum presented here was taken with the east-west slit placed along the northern part of the shell, but [O III] $\lambda 4363$ was not detected. The resulting (bright-line) oxygen abundance $(12+\log (\mathrm{O} / \mathrm{H})=7.97)$ is the first ever published for this dwarf. The adopted nitrogenand neon-to-oxygen ratios are: $\log (\mathrm{N} / \mathrm{O})=-1.37$, and $\log (\mathrm{Ne} / \mathrm{O})=-0.21$, respectively. The derived $\mathrm{Ne} / \mathrm{O}$ ratio is somewhat large compared to known values. However, the $I([\mathrm{Ne}$ III] $] 33869) / I(\mathrm{H} \beta)$ ratio is comparable to that observed in the nearby dI IC 4662 in the Local Volume (Heydari-Malayeri et al. 1990). Taking their published intensity ratios and temperatures for IC 4662, the derived value of $\log (\mathrm{Ne} / \mathrm{O})$ would be about zero.

\subsubsection{IC 5152}

Recent attention has been paid to this field dI, although a very bright foreground star in the northwest corner of the galaxy prevents deep imaging from taking place. Talent (1980) and Webster et al. (1983) obtained spectroscopy of the brightest H II region. Zijlstra \& Minniti (1999) obtained deep stellar photometry and constructed colour-magnitude diagrams; their field included the southeast quadrant of the galaxy to avoid the bright foreground star. A young population is indicated by the presence of H II regions and ultraviolet bright stars. A distance was inferred after comparison with colour-magnitude diagrams of other similar dIs and with theoretical isochrones. For the intermediate-age stars a metallicity of one-tenth solar was inferred. With resolved photometry from the HST,
Karachentsev et al. (2002c) have recently derived a TRGB distance of $2.07 \mathrm{Mpc}$.

The brightest $\mathrm{H}$ II region is located at the northeast corner of the dwarf and is labelled H II region \#A by Webster et al. (1983); this HII region is believed to be the same one measured by Talent (1980). Our spectrum of H II region \#A is the most up-to-date since the work of Talent (1980) and Webster et al. (1983). Talent (1980) measured [O III] $\lambda 4363$ and obtained an oxygen abundance of $12+\log (\mathrm{O} / \mathrm{H})=8.36$ and $\log (\mathrm{N} / \mathrm{O})=-1.52$. Webster et al. (1983) derived a brightline abundance of $12+\log (\mathrm{O} / \mathrm{H})=8.35$. We obtained an [O III] $\lambda 4363$ measurement of H II region \#A and the resulting oxygen abundance is $12+\log (\mathrm{O} / \mathrm{H})=7.92 \pm 0.07$. Our value of the oxygen abundance is about one-tenth of the solar value, which is consistent with the stellar metallicity inferred by Zijlstra \& Minniti (1999), but 2.7 times lower than the values reported by Talent and Webster et al. Our reported value of $\log (\mathrm{N} / \mathrm{O})=-1.05 \pm 0.12$ is three times larger than the value given by Talent (1980). Also, our adopted neon-to-oxygen ratio is $\log (\mathrm{Ne} / \mathrm{O})=-0.69 \pm 0.08$, which is in rough agreement with determinations by Izotov \& Thuan (1999) for other dwarf galaxies at similar oxygen abundance.

Additional spectra have been obtained of an H II region in the southwest region of the galaxy by Hidalgo-Gámez \& Olofsson (2002). They measured [O III] $\lambda 4363$ for which they derived an oxygen abundance of $12+\log (\mathrm{O} / \mathrm{H})=8.2$, although their corrected $I(\mathrm{H} \alpha) / I(\mathrm{H} \beta)$ ratio is over a factor of two lower than the theoretical value for typical H II regions. Future observations of this and other H II regions would be very helpful to confirm the nature of the unusual H II region observed by Hidalgo-Gámez \& Olofsson, the relatively large N/O, and the homogeneity of oxygen abundances in this dwarf galaxy. The ground-based stellar photometry obtained by Zijlstra \& Minniti (1999) includes the centre and the southeast portion of the dwarf galaxy, which unfortunately avoids the part of the galaxy where H II region \#A is located. Deeper resolved photometry would be very useful in gaining further clues about the underlying stellar populations and determining the history of star formation.

\subsubsection{NGC 2915}

This galaxy is likely a relatively nearby blue compact dwarf galaxy. Sérsic et al. (1977) presented one of the first comprehensive studies using photoelectric photometry, spectrograms, and radio observations. With updated broadband photometry, Meurer et al. (1994) found that the dwarf contained two dominant stellar populations. Current star formation is occurring at the centre where most of the ionized gas and a bright blue population of stars are present. A red diffuse population with an exponential surface brightness profile lies outside of the central region. Meurer et al. (1994) label this galaxy as an amorphous blue compact dwarf galaxy with properties of a dwarf elliptical at large galactocentric radii. Meurer et al. (1996) obtained H I synthesis observations and found that the H I is five times larger in spatial extent than the optical extent defined by the Holmberg radius. The galaxy also has a central bar and 
spiral arms with a maximum rotation speed of $v_{\max } \simeq$ $85 \mathrm{~km} \mathrm{~s}^{-1}$. Their subsequent modelling shows that the dark matter content is dominant at all radii. Bureau et al. (1999) studied the barred spiral arm structure and found that a rotating triaxial dark matter halo can best explain the $\mathrm{H} \mathrm{I}$ observations.

The observed spectrum presented here was taken with the east-west slit placement through the centre of the galaxy. Our adopted (average) value of the oxygen abundance is $12+\log (\mathrm{O} / \mathrm{H})=8.30$. The adopted (average) nitrogen-tooxygen ratio is $\log (\mathrm{N} / \mathrm{O})=-1.48$. From the spectrum of the galaxy nucleus measured by Sérsic et al. (1977), they found $I(\mathrm{H} \alpha) / I([\mathrm{O} \mathrm{III}] \lambda \lambda 4959,5007)=0.25$ and $[I(\mathrm{H} \alpha)+$ $I([\mathrm{~N} \mathrm{II}] \lambda 6583)] / I([\mathrm{~S} \mathrm{II}] \lambda \lambda 6716,6731)=5$, which they claimed as evidence for a low excitation H II region. Our corresponding ratios are: $I(\mathrm{H} \alpha) / I([\mathrm{O} \mathrm{III}] \lambda \lambda 4959,5007)=1.2$, and $[I(\mathrm{H} \alpha)+I([\mathrm{~N} \mathrm{II}] \lambda 6583)] / I([\mathrm{~S} \mathrm{II}] \lambda \lambda 6716,6731)=5.1$. Meurer et al. (1994) also obtained a nuclear spectrum and derived an upper limit to the oxygen abundance, $12+\log (\mathrm{O} / \mathrm{H}) \lessgtr 8.5$, which is consistent with our measurements.

\subsubsection{NGC 3109}

NGC 3109 is the most massive galaxy in the nearby, extremely poor Antlia-Sextans group (van den Bergh 1999; Tully et al. 2002). While considered as dI by some workers, this galaxy may be better described as a Magellanic spiral or, perhaps, even a dwarf spiral (Grebel 2001a,b). Studies of the H I gas and stellar content have been well documented for this galaxy. For example, Carignan (1985) and Jobin \& Carignan (1990) obtained optical and H I observations and showed that: (1) compared to similar galaxies (e.g., SMC), the total galaxy luminosity is comparable, but the optical extent is roughly two times larger; (2) the outer parts of the H I gas is warped, likely due to an interaction with the Antlia dwarf galaxy; (3) model fits to the rotation profile show that there is roughly ten times more dark matter than luminous matter; and (4) that the gas is a good tracer of the dark matter distribution. Musella et al. (1997) and Minniti et al. (1999) have obtained distances of $1.36 \mathrm{Mpc}$ and 1.33 Mpc using observations of Cepheid variable stars and the tip of the red giant branch, respectively. Also, Karachentsev et al. (2002c) obtained a distance of $1.33 \mathrm{Mpc}$ from the magnitude of the tip of the red giant branch using HST WFPC2 observations. Grebel et al. (2003) have obtained $[\mathrm{Fe} / \mathrm{H}] \simeq-1.7 \mathrm{dex}$ for the metallicity of the red giant branch, which points to the metal-poor nature of this galaxy.

Richer \& McCall (1992) identified a number of H II regions and planetary nebulae in the eastern section of the galaxy. M. McCall and C. Stevenson carried out spectroscopic measurements at the Steward Observatory of H II region "\#5"; see Richer \& McCall (1992) for their labelling. An [O III] $\lambda 4363$ detection was reported and a lower branch oxygen abundance was obtained (7.73) by Lee et al. (2003a). For the present work, measurements were carried out for H II region "\#6" (as labelled by Richer \& McCall 1992). Using $I([\mathrm{~N} \mathrm{II}] \lambda 6583) / I(\mathrm{H} \alpha)$ and the calibrations by van Zee et al. (1998b) and Denicoló et al. (2002), the resulting oxygen abundances are consistent with lower branch values, but are $\approx 0.4$ dex higher than the values determined from either the McGaugh or the Pilyugin calibration. We adopt here the McGaugh and Pilyugin calibrations, as most of the remaining bright-line oxygen abundances in the southern sample are derived in a similar manner. So, taking the average of the ten values listed in Table 9, the adopted oxygen abundance is $12+\log (\mathrm{O} / \mathrm{H})=7.63$, which is similar to the value (7.73) determined by Lee et al. (2003a).

\subsubsection{NGC 5264}

NGC 5264 is a Cen A dwarf galaxy with a TRGB distance of $4.53 \mathrm{Mpc}$ (Karachentsev et al. 2002b). Compared to other HII spectra, our spectrum shows relatively low $I([\mathrm{O} \mathrm{III}] \lambda 5007) / I(\mathrm{H} \beta), \quad$ whereas $\quad I([\mathrm{O} \mathrm{II}] \lambda 3727) / I(\mathrm{H} \beta)$, $I([\mathrm{~N} \mathrm{II}] \lambda 6583) / I(\mathrm{H} \alpha)$, and $I([\mathrm{~S} \mathrm{II}] \lambda \lambda 6716,6731) / I(\mathrm{H} \alpha)$ are high. The $[\mathrm{N} \mathrm{II}] /[\mathrm{O} \mathrm{II}]$ discriminant indicates an upper branch abundance. Because of the low negative value $(-0.599)$ for $\log O_{32}$, the oxygen abundance derived using the Pilyugin bright-line method is somewhat uncertain, as his method is calibrated to observations of $\mathrm{HII}$ regions with larger values of $\log O_{32}$. Using $I([\mathrm{~N} \mathrm{II}] \lambda 6583) / I(\mathrm{H} \alpha)$, the calibrations of van Zee et al. (1998b) and Denicoló et al. (2002) give $12+\log (\mathrm{O} / \mathrm{H})=8.68$ and 8.55 , respectively, which agree with the upper branch abundances from the McGaugh and the Pilyugin calibrations. Taking an average of the values from each calibration, the adopted oxygen abundance is $12+\log (\mathrm{O} / \mathrm{H})=8.61$, which is similar to measured abundances in spiral galaxies. Using the Thurston et al. (1996) method for $\mathrm{HII}$ regions in spiral galaxies, the nitrogen-to-oxygen abundance ratio is $\log (\mathrm{N} / \mathrm{O})=-0.57$.

NGC 5264 has a higher oxygen abundance and a nitrogento-oxygen ratio than expected for its galaxy luminosity. This galaxy may be an example of a dwarf spiral (e.g., Schombert et al. 1995), whose luminosity is similar to the SMC, but whose oxygen abundance is a factor of four larger than the SMC. A systematic spectroscopic survey of H II regions in NGC 5264 should show whether or not the galaxy has a radial gradient in oxygen abundance.

Compared to typical dIs, the $M_{\mathrm{HI}} / L_{B}$ value for NGC 5264 is somewhat low compared to other late-type galaxies of similar luminosity. NGC 5264 is located one degree to the east of the luminous spiral NGC 5236 (M 83), where an interaction with the latter could remove HI gas from NGC 5264. Interestingly, there is in the vicinity another Cen A group member dI, UGCA 365 (ESO444-G078), which appears to be even closer in projection to M 83 (see Fig. 1 in Karachentsev et al. 2002b). There may be an interaction between M 83, UGCA 365, and NGC 5264, which could explain the comparatively lower $\mathrm{HI}$ content in the latter galaxy.

\subsubsection{Sag DIG}

The Sagittarius dwarf irregular (Sag DIG) galaxy was first discovered by Cesarsky et al. (1977) and later confirmed by Longmore et al. (1978). Strobel et al. (1991) detected what appeared to be three HII regions; only one appears to be a true HII region, whereas the other two sources are likely stars in 
H $\alpha$ emission. Young \& Lo (1997) found that the H I component was more spatially extended than the stellar component. An H I clump of relatively high density is nearly spatially coincident with the H II region. There is no clear rotational motion for the HI gas. Rather, the gas appears to be supported by random motions with a broad- and a narrow-velocity component. Using resolved stellar photometry, Karachentsev et al. (1999) and Lee \& Kim (2000) independently obtained TRGB distances of $1.11 \mathrm{Mpc}$, which places this dwarf at the outer boundaries of the Local Group and makes the membership to the Local Group uncertain. In addition, photometry has revealed that the underlying stellar population is very metal-poor with $[\mathrm{Fe} / \mathrm{H}] \lessgtr-2$, which was also noted by Momany et al. (2002).

Skillman et al. (1989b) attempted deep spectroscopy, but failed to detect [O III] $\lambda 4363$ or [N II] 26583 . Saviane et al. (2002) have also recently obtained deep emission-line spectroscopy of the H II region. Despite three hours of total exposure, [O III] $\lambda 4363$ was not detected. Although Saviane et al. (2002) used a larger telescope, they did not detect [O III] $\lambda 4363$. The oxygen abundance derived here $(12+\log (\mathrm{O} / \mathrm{H})=7.39)$ agrees with the bright-line abundance obtained by Skillman et al. (1989b) and with the upper end of the range of brightline abundances derived by Saviane et al. (2002). The oxygenpoor interstellar medium is consistent with the very low iron abundance seen in the stars. Although $\approx 0.2$ dex lower than the value obtained by Saviane et al. (2002), our derived $\log (\mathrm{N} / \mathrm{O})=-1.63$ is almost identical with the near-constant value for blue compact dwarf galaxies (Izotov \& Thuan 1999), even though Sag DIG is a very low-luminosity dI with fairly quiescent star formation. However, the $M_{\mathrm{HI}} / L_{B}$ value and the relatively low N/O may be indicative of a very recent (and, perhaps, small) burst, which is borne out by the young stars seen in resolved photometry (Lee \& Kim 2000).

\subsubsection{UGCA 442}

Miller (1996) obtained $\mathrm{H} \alpha$ imaging of this edge-on galaxy in the Scl group and found five H II regions. Spectroscopy for two H II regions in the southwest part of the disk (\#2 and \#4 as labelled by Miller 1996) did not reveal [O III] $\lambda 4363$; he derived an average bright-line oxygen abundance of $12+\log (\mathrm{O} / \mathrm{H})=7.90$. These two $\mathrm{H}$ II regions appear to be in a part of the galaxy where Côté et al. (2000) found a local maximum in H I; the HI extent is about a factor of five larger than the optical extent. Our spectrum of H II region \#2 (labelling by Miller 1996) also did not reveal [O III] $\lambda 4363$, and our subsequent bright-line oxygen abundance $(12+\log (\mathrm{O} / \mathrm{H})=7.85)$ agrees with the value obtained by Miller (1996). The adopted nitrogen-to-oxygen ratio is $\log (\mathrm{N} / \mathrm{O})=-1.41$. The $M_{\mathrm{HI}} / L_{B}$ value for this dwarf is comparable to typical dIs at the given luminosity.

\subsection{Metallicity-luminosity relation}

Skillman et al. (1989a) and Richer \& McCall (1995) showed for dIs that metallicity in the form of oxygen abundances

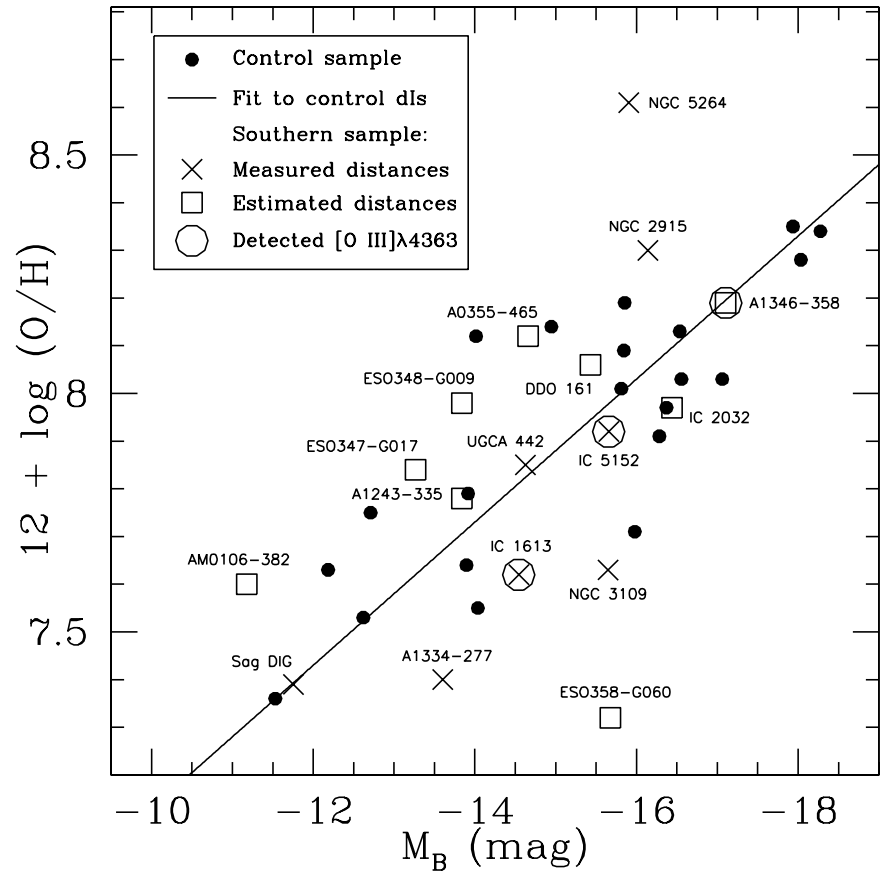

Fig. 6. Oxygen abundance versus galaxy luminosity in $B$. Filled circles mark the control sample of dIs (Lee et al. 2003a). The best-fit to the control sample is shown as a solid line. For the present sample of southern dwarfs, crosses and open squares mark galaxies for which distances are measured and estimated, respectively. In addition, galaxies for which [O III] $\lambda 4363$ has been measured are marked with open circles. For galaxies with [O III] $\lambda 4363$ measurements, the oxygen abundance has an uncertainty of at most 0.1 dex. The remaining galaxies without [O III] $\lambda 4363$ measurements have an uncertainty of 0.2 dex in oxygen abundance. A typical uncertainty of 0.2 mag in absolute magnitude accounts for the various methods used to determine distances for dIs in both samples.

increase with the galaxy luminosity in $B$. This relationship has been interpreted as being representative of a relationship between metallicity and mass, at least where stellar mass is concerned. The metallicity-luminosity diagram is shown in Fig. 6. Plotted are the set of dwarfs in the control sample from Lee et al. (2003a). A new fit to galaxies with [O III] $\lambda 4363$ abundances and well-measured distances (i.e., control sample excluding NGC 3109, and new data for IC 1613 and IC 5152) leaves the slope and intercept essentially unchanged within the stated errors compared to the values obtained in the fits by Richer \& McCall (1995) and Lee et al. (2003a).

Plotted also are dwarfs from the southern sample, combining oxygen abundances derived using the bright-line method and both measured and estimated distances. Most dIs in the present sample have oxygen abundances consistent with the metallicity-luminosity relation defined by dIs in the control sample. Because of the excellent agreement with the metallicity-luminosity relation, IC 5152 will in the future be added to the growing list of galaxies in the control sample with [O III] $\lambda 4363$ measurements and distances derived from resolved stellar photometry (see Sect. 2.2).

There may be an impression that the metallicity-luminosity relation exhibits greater scatter at the range of luminosities shown. At lower galaxy luminosities, Sag DIG and A1334-277 
have measured distances, but one should keep in mind that their oxygen abundances were obtained using the bright-line method. ESO358-G060 is an outlier, which appears to be too bright in $B$ luminosity for its low oxygen abundance. If the oxygen abundance is confirmed, this galaxy would resemble a blue compact dwarf galaxy, as galaxies representative of this type lie mostly below the metallicity-luminosity relation (e.g., Kunth \& Östlin 2000). At $M_{B} \approx-16$, the adopted oxygen abundance for NGC 5264 (Sect. 6.1.16) is approximately 0.2 to 0.3 dex higher than expected at the given luminosity. Additional H II region spectra would be useful in confirming this result.

\subsection{Relative nitrogen to oxygen abundances}

A plot of $\log (\mathrm{N} / \mathrm{O})$ versus $\log (\mathrm{O} / \mathrm{H})$ is shown for star-forming dwarf galaxies in Fig. 7. In general, the present data overlaps with the loci defined by dwarfs in the control sample (Lee et al. 2003a) and by other dwarf galaxies (Garnett 1990; Kobulnicky \& Skillman 1996; van Zee et al. 1997; Izotov \& Thuan 1999), although there are a few HII regions with elevated values of $\log (\mathrm{N} / \mathrm{O})$ for their oxygen abundance. There still appears to be a great deal of dispersion in $\mathrm{N} / \mathrm{O}$ values at a given oxygen abundance. For IC $1613 \mathrm{H} \mathrm{II \# 37} \mathrm{and} \mathrm{IC} 5152 \mathrm{H} \mathrm{II \# A,} \mathrm{their} \log (\mathrm{N} / \mathrm{O})$ values are somewhat elevated compared to the mean value for H II from dIs in the control sample. For NGC 5264, the oxygen and nitrogen abundances are in better agreement with values found in spiral galaxies; see the compilation by Henry et al. (2000).

\section{Conclusions}

Optical emission-line spectroscopy of H II regions was obtained in dwarf irregular galaxies in the Centaurus A group, the Sculptor group, the Antlia-Sextans group, the Local Group, and in the field. [O III] $\lambda 4363$ was measured in A1346-358, IC 1613, and IC 5152, and the standard method was used to obtain oxygen abundances. For the remaining galaxies, the brightline method was used to compute oxygen abundances with the McGaugh and Pilyugin calibrations. The [N II]/[O II] intensity ratio was used to break the degeneracy in the bright-line method and applied to both calibrations. For NGC 3109 and $\mathrm{NGC} 5264$, the $[\mathrm{N} \mathrm{II}] / \mathrm{H} \alpha$ intensity ratio was also used to confirm their abundances. ESO358-G030 has the lowest oxygen abundance in the sample with a value of $12+\log (\mathrm{O} / \mathrm{H})=7.32$, which is comparable to the value for the second most metalpoor galaxy known (SBS 0335-052).

Oxygen abundances for dwarfs in the southern sample agree with the metallicity-luminosity relationship defined by dwarf irregulars in the control sample. Three dwarfs with direct abundances are found to be in very good agreement with the relation, whereas the remaining galaxies with bright-line abundances are in comparable agreement with larger scatter. NGC 5264 appears to have an oxygen abundance approximately two times larger than expected for its luminosity. Extensive spectroscopy of H II regions in NGC 5264 will confirm whether the galaxy is a dwarf irregular galaxy with a zero or very small abundance gradient or a low-luminosity spiral galaxy with a significant abundance gradient.

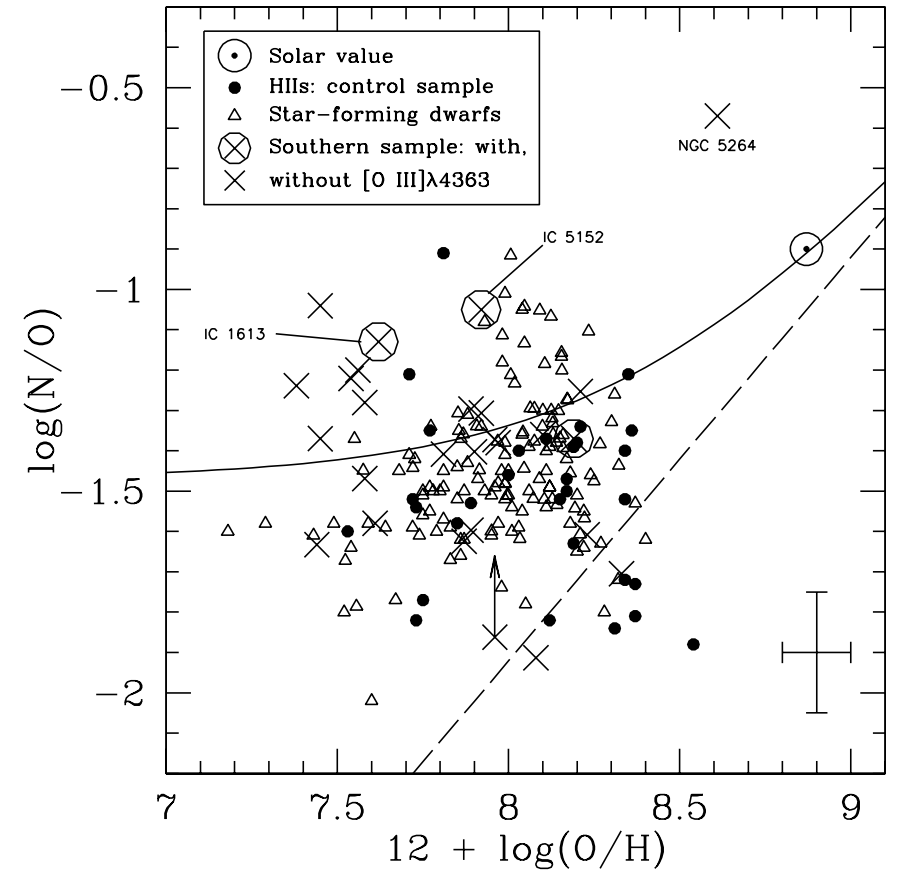

Fig. 7. Nitrogen-to-oxygen abundance ratio versus oxygen abundance. Filled circles indicate H II regions from the control sample of dIs, whose oxygen abundances were obtained directly from measurements of the [O III] $\lambda 4363$ emission line. Open triangles represent other starforming dwarf galaxies from Garnett (1990), Kobulnicky \& Skillman (1996), van Zee et al. (1997), and Izotov \& Thuan (1999). Crosses mark HII regions from dwarfs in the southern sample; open circles denote galaxies where [O III] $\lambda 4363$ was detected. Labelled are IC 1613 H II\#37 and IC 5152 H II\#A for their unusually high N/O ratios. Two models for the production of nitrogen are shown (VilaCostas \& Edmunds 1993); the solid line is a model with both primary and secondary production, whereas the dashed line is a model for secondary production. The solar value of N/O (Grevesse et al. 1996) is also indicated. Typical errors in $\log (\mathrm{O} / \mathrm{H})$ and $\log (\mathrm{N} / \mathrm{O})$ are shown at the lower right.

Nitrogen-to-oxygen abundance ratios for dwarfs in the southern sample are comparable to those in the control sample at a given oxygen abundance, although IC 1613 and IC 5152 have larger N/O values compared to nearby dwarf irregulars at a given oxygen abundance. Additional stellar photometry for IC 5152 should provide further clues to the star formation and enrichment history of this nearby dwarf.

Acknowledgements. We are grateful to the anonymous referee for improving the presentation of the manuscript, and to the CTIO staff for their help with the observations. Partial support for this work was provided by NASA through grant GO-08192.97A from the Space Telescope Science Institute, which is operated by the Association of Universities for Research in Astronomy, Inc., under NASA contract NAS5-26555. H. L. acknowledges MPIA for their financial support, and Stephanie Côté and Evan Skillman for discussions and making available a copy of their paper before publication. Some data were accessed as Guest User, Canadian Astronomy Data Center, which is operated by the Dominion Astrophysical Observatory for the National Research Council of Canada's Herzberg Institute of Astrophysics. This research has made use of the NASA/IPAC Extragalactic Database (NED), which is operated by the Jet Propulsion Laboratory, California 
Institute of Technology, under contract with the National Aeronautics and Space Administration.

\section{Appendix A: Other measurements}

Skillman et al. (2003) have obtained emission-line spectroscopy of late-type dwarf galaxies in the Sculptor group with the 4-m telescope at CTIO. Their sample included ESO347-G017, ESO348-G009, and UGCA 442; they measured [O III] $\lambda 4363$ in ESO347-G017 and UGCA 442. Our bright-line oxygen abundances for ESO347-G017, ESO348-G009, and UGCA 442 agree with the abundances obtained by Skillman et al.

\section{References}

Allende Prieto, C., Lambert, D. L., \& Asplund, M. 2001, ApJ, 556, L63

Barnes, D. G., \& de Blok, W. J. G. 2001, AJ, 122, 825

Barnes, D. G., Staveley-Smith, L., Webster, R. L., \& Walsh, W. 1997, MNRAS, 288, 307

Bureau, M., Freeman, K. C., Pfitzner, D. W., \& Meurer, G. R. 1999, AJ, 118, 2158

Carignan, C. 1985, ApJ, 299, 59

Carrasco, E. R., de Oliveira, C. M. Infante, L., \& Bolte, M. 2001, AJ, 121,148

Cesarsky, D. A., Lequeux, J., Laustsen, S., Schuster, H.-E., \& West, R. M. 1977, A\&A, 61, L31 (erratum: 1978, A\&A, 65, 153)

Cole, A. A., Tolstoy, E., Gallagher, J. S., et al. 1999, AJ, 118, 1657

Côté, S., Freeman, K. C., Carignan, C., \& Quinn, P. J. 1997, AJ, 114, 1313

Côté, S., Carignan, C., \& Freeman, K. C. 2000, AJ, 120, 3027

Davidson, K., \& Kinman, T. D. 1982, PASP, 94, 634

Denicoló, G., Terlevich, R., \& Terlevich, E. 2002, MNRAS, 330, 69

Dinerstein, H. L. 1990, in The Interstellar Medium in Galaxies, ed. H. A. Thronson, \& J. M. Shull (Dordrecht: Kluwer), 257

D’Odorico, S., \& Dopita, M. 1983, in Supernova Remnants and their X-Ray Emission, ed. J. Danziger, \& P. Gorenstein (Dordrecht: Kluwer), IAU Symp., 101, 517

D’Odorico, S., \& Rosa, M. 1982, A\&A, 105, 410

D’Odorico, S., Dopita, M., \& Benvenuti, P. 1980, A\&AS, 40, 67

Dolphin, A. E., Makarova, L., Karachentsev, I. D., et al. 2001a, MNRAS, 324, 249

Dolphin, A. E., Saha, A., Skillman, E. D., et al. 2001b, ApJ, 550, 554

Drinkwater, M. J., Gregg, M. D., Holman, B. A., \& Brown, M. J. I. 2001, MNRAS, 326, 1076

Feitzinger, J. V., \& Galinski, Th. 1985, A\&AS, 61, 503

Ferguson, H. C. 1989, AJ, 98, 367

Garcia, A. M. 1993, A\&AS, 100, 47

Garnett, D. R. 1990, ApJ, 363, 142

Grebel, E. K. 1999, in The Stellar Content of the Local Group, ed. P. Whitelock, \& R. Cannon (San Francisco: Astron. Soc. of the Pacific), IAU Symp., 192, 17

Grebel, E. K. 2000, in Star Formation from the Small to the Large Scale, ESA SP-445, ed. F. Favata, A. A. Kaas, \& A. Wilson (Noordwijk: ESA), 87

Grebel, E. K. 2001a, Ap\&SS, 277, 231

Grebel, E. K. 2001b, in Dwarf Galaxies and the Environment, ed. K. S. De Boer, R.-J. Dettmar, \& U. Klein (Aachen: Shaker Verlag), 45

Grebel, E. K., Seitzer, P., Dolphin, A., et al. 2000, in Stars, Gas, and Dust in Galaxies: Exploring the Links, ed. D. Alloin, K. Olsen, \& G. Galaz (San Francisco: Astron. Soc. of the Pacific), ASP Conf. Ser., 221, 147
Grebel, E. K., Gallagher, J. S., \& Harbeck, D. R. 2003, AJ, 125, in press [astro-ph/0301025]

Grevesse, N., Noels, A., \& Sauval, A. J. 1996, in Cosmic Abundances: Proceedings of the 6th Annual October Astrophysics Conference, ed. S. S. Holt, \& G. Sonneborn (San Francisco: Astron. Soc. of the Pacific), ASP Conf. Ser., 99, 117

Henry, R. B. C., Edmunds, M. G., \& Köppen, J. 2000, ApJ, 541, 660

Heydari-Malayeri, M., Melnick, J., \& Martin, J.-M. 1990, A\&A, 234, 99

Hidalgo-Gámez, A. M., \& Olofsson, K. 2002, A\&A, 389, 836

Hidalgo-Gámez, A. M., Masegosa, J., \& Olofsson, K. 2001, A\&A, 369,797

Hodge, P., Lee, M. G., \& Gurwell, M. 1990, PASP, 102, 1245

Hoffman, G. L., Salpeter, E. E., Farhat, B., et al. 1996, ApJS, 105, 269

Hoffman, G. L., Lu, N. Y., Salpeter, E. E., \& Connell, B. M. 1999, AJ, 117,811

Holweger, H. 2001, in Solar and Galactic Composition, ed. R. F. Wimmer-Schweingruber (Berlin: Springer), 23

Huchtmeier, W. K., \& Richter, O. G. 1986, A\&A, 63, 323

Huchtmeier, W. K., Karachentsev, I. D., Karachentseva, V. E., \& Ehle, M. 2000a, A\&AS, 141, 469

Huchtmeier, W. K., Karachentsev, I. D., \& Karachentseva, V. E. 2000b, A\&AS, 147, 487

Hunter, D. A., \& Gallagher, J. S. 1985, ApJS, 58, 533

Izotov, Y. I., \& Thuan, T. X. 1999, ApJ, 511, 639

Izotov, Y. I., \& Thuan, T. X. 2002, ApJ, 567, 875

Izotov, Y. I., Thuan, T. X., \& Lipovetsky, V. A. 1994, ApJ, 435, 647

Izotov, Y. I., Lipovetsky, V. A., Chaffee, F. H., et al. 1997a, ApJ, 476, 698

Izotov, Y. I., Thuan, T. X., \& Lipovetsky, V. A. 1997b, ApJS, 108, 1

Jobin, M., \& Carignan, C. 1990, AJ, 100, 648

Karachentseva, V. E., \& Karachentsev, I. D. 1998, A\&AS, 127, 409

Karachentsev, I. D., Karachentseva, V. E., \& Mineva, V. A. 1981, Soviet Astron. Lett., 7, 298

Karachentsev, I. D., Aparicio, A., \& Makarova, L. 1999, A\&A, 352, 363

Karachentsev, I. D., Karachentseva, V. E., Suchkov, A. A., \& Grebel, E. K. 2000, A\&AS, 145, 415

Karachentsev, I. D., Dolphin, A. E., Geisler, D., et al. 2002a, A\&A, 383,125

Karachentsev, I. D., Sharina, M. E., Dolphin, A. E., et al. 2002b, A\&A, 385,21

Karachentsev, I. D., Sharina, M. E., Makarov, D. I., et al. 2002c, A\&A, 389,812

Karachentsev, I. D., Makarov, D. I., Sharina, M. E., et al. 2003a, A\&A 398, 479

Karachentsev, I. D., Grebel, E. K., Sharina, M. E., et al. 2003b, A\&A, accepted [astro-ph/0302045]

Kingsburgh, R. L., \& Barlow, M. J. 1995, A\&A, 295, 171

Kobulnicky, H. A., \& Skillman, E. D. 1996, ApJ, 471, 211

Krawchuk, C., McCall, M. L., Komljenovic, M., et al. 1997, in Planetary Nebulae, ed. H. J. Habing, \& H. J. G. L. M. Lamers (Dordrecht: Kluwer), IAU Symp., 180, 116

Kunth, D., \& Östlin, G. 2000, A\&ARv, 10, 1

Lee, H. 2001, Ph.D. Thesis, York University

Lee, H., McCall, M. L., Kingsburgh, R., Ross, R., \& Stevenson, C. C. 2003a, AJ, 125, 146

Lee, H., McCall, M. L., \& Richer, M. G. 2003b, AJ, submitted

Lee, M. G., \& Kim, S. C. 2000, AJ, 119, 777

Lequeux, J., Meyssonnier, N., \& Azzopardi, M. 1987, A\&AS, 67, 169

Longmore, A. J., Hawarden, T. G., Webster, B. L., et al. 1978, MNRAS, 183, 97P

Longmore, A. J., Hawarden, T. G., Goss, W. M., Mebold, U., \& Webster, B. L. 1982, MNRAS, 200, 325 
Makarova, L. N., Karachentsev, I. D., Grebel, E. K., \& Barsunova, O. Yu. 2002, A\&A, 384, 72

Mateo, M. 1998, ARA\&A, 36, 435

Matthews, L. D., \& Gallagher, J. S. 2002, ApJS, 141, 429

Matthews, L. D., van Driel, W., \& Gallagher, J. S. 1998, AJ, 116, 1169

Mayer, L., Governato, F., Colpi, M., et al. 2001, ApJ, 547, L123

McCall, M. L., Rybski, P. M., \& Shields, G. A. 1985, ApJS, 57, 1

McGaugh, S. S. 1991, ApJ, 380, 140

McGaugh, S. S. 1994, ApJ, 426, 135

Melnick, J., Heydari-Malayeri, M., \& Leisy, P. 1992, A\&A, 253, 16

Meurer, G. R., Mackie, G., \& Carignan, C. 1994, AJ, 107, 2021

Meurer, G. R., Carignan, C., Beaulieu, S. F., \& Freeman, K. C. 1996, $\mathrm{AJ}, 111,1551$

Miller, B. W. 1996, AJ, 112, 991

Minniti, D., Zijlstra, A. A., \& Alonso, M. V. 1999, AJ, 117, 881

Momany, Y., Held, E. V., Saviane, I., \& Rizzi, L. 2002, A\&A, 390, 59

Mould, J. R., Hughes, S. M. G., Stetson, P. B., et al. 2000, ApJ, 528, 655

Musella, I., Piotto, G., \& Capaccioli, M. 1997, AJ, 114, 976

Osterbrock, D. E. 1989, Astrophysics of Gaseous Nebulae and Active Galactic Nuclei (Mill Valley: University Science Books)

Pagel, B. E. J., Edmunds, M. G., Blackwell, D. E., et al. 1979, MNRAS, 189, 95

Pagel, B. E. J., Simonson, E. A., Terlevich, R. J., \& Edmunds, M. G. 1992, MNRAS, 255, 325

Peimbert, M., Bohigas, J., \& Torres-Peimbert, S. 1988, Rev. Mex. Astron. Astrofis., 16, 45

Pildis, R. A., Schombert, J. M., \& Eder, J. A. 1997, ApJ, 481, 157

Pilyugin, L. S. 2000, A\&A, 362, 325

Pilyugin, L. S. 2001a, A\&A, 369, 594

Pilyugin, L. S. 2001b, A\&A, 374, 412

Price, J. S., Mason, S. F., \& Gullixson, C. A. 1990, AJ, 100, 420

Richer, M. G., \& McCall, M. L. 1992, AJ, 103, 54

Richer, M. G., \& McCall, M. L. 1995, ApJ, 445, 642

Roberts, M. S., \& Haynes, M. P. 1994, ARA\&A, 32, 115

Rosado, M., Valdez-Gutiérrez, M., Georgiev, L., et al. 2001, AJ, 122 , 194

Sandage, A. R. 1971, ApJ, 166, 13

Saviane, I., Rizzi, L., Held, E. V., Bresolin, F., \& Momany, Y. 2002, A\&A, 390, 59
Schombert, J. M., Pildis, R. A., Eder, J. A., \& Oemler, A., Jr. 1995, AJ, 110, 2067

Schröder, A., Drinkwater, M. J., \& Richter, O.-G. 2001, A\&A, 376, 91

Sérsic, J. L., Bajaja, E., \& Colomb, R. 1977, A\&A, 59, 19

Skillman, E. D. 1996, in The Minnesota Lectures on Extragalactic Neutral Hydrogen (San Francisco: Astron. Soc. of the Pacific), ASP Conf. Ser., 106, 208

Skillman, E. D., Kennicutt, R. C., Jr., \& Hodge, P. 1989a, ApJ, 347, 875

Skillman, E. D., Terlevich, R., \& Melnick, J. 1989b, MNRAS, 240, 563

Skillman, E. D., Terlevich, R. J., Kennicutt, R. C., Jr., et al. 1994, ApJ, 431, 172

Skillman, E. D., Bomans, D. J., \& Kobulnicky, H. A. 1997, ApJ, 474, 205

Skillman, E. D., Côté, S., \& Miller, B. W. 2003, AJ, 125, 610

Staveley-Smith, L., Davies, R. D., \& Kinman, T. D. 1992, MNRAS, 258,334

Strobel, N. V., Hodge, P., \& Kennicutt, R. C., Jr. 1991, ApJ, 383, 148

Talent, D. L. 1980, Ph.D. Thesis, Rice University

Thurston, T. R., Edmunds, M. G., \& Henry, R. B. C. 1996, MNRAS, 283, 990

Tully, R. B., Somerville, R. S., Trentham, N., \& Verheijen, M. A. W. 2002, ApJ, 569, 573

Valdez-Gutiérrez, M., Rosado, M., Georgiev, L., et al. 2001, A\&A, 366,35

van den Bergh, S. 1999, ApJ, 517, L97

van Zee, L., Haynes, M. P., \& Salzer, J. J. 1997, AJ, 114, 2479

van Zee, L., Skillman, E. D., \& Salzer, J. J. 1998a, AJ, 116, 1186

van Zee, L., Salzer, J. J., Haynes, M. P., O’Donoghue, A. A., \& Balonek, T. J. 1998b, AJ, 116, 2805

Vila-Costas, M. B., \& Edmunds, M. G. 1993, MNRAS, 265, 199

Webster, B. L., \& Smith, M. G. 1983, MNRAS, 204, 743

Webster, B. L., Longmore, A. J., Hawarden, T. G., \& Mebold, U. 1983, MNRAS, 205, 643

Young, L. M., \& Lo, K. Y. 1997, ApJ, 490, 710

Zijlstra, A. A., \& Minniti, D. 1999, AJ, 117, 1743 